\title{
THE ELLIPSOIDAL CAVITY IN THE PRESENCE OF A LOW-FREQUENCY ELASTIC WAVE*
}

\author{
BY \\ GEORGE DASSIOS (University of Patras) \\ AND \\ KIRIAKIE KIRIAKI ( National Technical University of Athens)
}

\begin{abstract}
We consider the problem of scattering of a longitudinal or a transverse plane elastic wave by a general ellipsoidal cavity in the low-frequency region. Explicit closed-form solutions for the zeroth- and first-order approximations are provided in terms of the physical and geometric characteristics of the scatterer, as well as the direction cosines of the incidence and observation points. This was made possible with the introduction of an analytical technique based on the Papkovich representations and their interdependence. The leading low-frequency term for the normalized spherical scattering amplitudes and the scattering cross section are also given explicitly. Degenerate ellipsoids corresponding to the prolate and oblate spheroids, the sphere, the needle, and the disc are considered as special cases.
\end{abstract}

1. Introduction. In [7] we gave a systematic analysis of the elastic scattering problem at low frequencies. We studied the four basic problems corresponding to either a longitudinal or a transverse incident wave which is scattered by a rigid body or a cavity consisting of a smooth, convex, and bounded three-dimensional set. In [8] we applied our method to the triaxial rigid ellipsoid, which is the most general second-degree geometric figure where the method of separation of variables and eigenfunction expansion can be applied. It turned out that the lack of rotational symmetry for the scatterer makes the problem very difficult to solve in closed analytical form, and a new calculational technique had to be introduced in order to find the first two low-frequency approximations in terms of ellipsoidal harmonics, Lamé functions, and standard elliptic integrals. The present work refers to the application of our general method to a triaxial cavity, thus completing our program of studying the theory and applications of fundamental elastic scattering problems. The technique used in [8] to evaluate the low-frequency coefficients for the rigid ellipsoid

\footnotetext{
* Received May 31, 1985.
} 
cannot be applied to the ellipsoidal cavity since the surface traction operator on the surface of the cavity complicates the boundary conditions. As a result, a more involved technique had to be elaborated in order to be able to find closed-form solutions in finite steps. These difficulties are expected since the surface traction operator involves not only the unit normal but also surface differential expressions which must be expressed in ellipsoidal curvilinear coordinates.

Ying and Truell [16] were the first to study the elastic scattering problem for longitudinal incidence on a sphere. The scattering problem for transverse incidence on a spherical cavity was investigated by Einspruch, Witterholt and Truell [9]. Lawrence [12], using an analytical method developed by Barratt and Collins [1], was able to evaluate the leading low-frequency term for the scattering cross section of an ellipsoid. Analytical work on the theory of scattering by ellipsoidal bodies can be found in $[2-4,6]$. The basic scattering theorems are given by Twersky in [14].

In Sec. 2 we formulate the two problems we discuss in this paper: the scattering of a longitudinal or a transverse wave by an ellipsoidal cavity. For completeness, we include all the necessary formulae, far-field expressions, integral representations, fundamental solutions, scattering amplitudes, and scattering cross sections. The wave problem is reduced to a sequence of elliptic problems which can be solved iteratively. The solution of these problems provides the corresponding low-frequency approximations of an appropriate expansion in powers of the wave number. By introducing Papkovich potentials, the solutions of all these elliptic problems are reduced to evaluation of the coefficients of appropriate orthogonal expansions of harmonic functions. To be able to evaluate these coefficients it is necessary to use the dependence of the vector and scalar Papkovich potentials to produce independent systems of linear equations with finite numbers of equations and unknowns. All the ellipsoidal harmonics, Lamé functions, and elliptic integrals used in this work are given in the Appendix (Sec. 8).

In Sec. 3 we apply our technique to solve the zeroth-order coefficient problem, while Sec. 4 contains the much more elaborate first-order coefficient of the low-frequency expansion. The final forms of these low-frequency approximations are expressed in a compact way that includes higher-order tensors, which describe the geometric and physical characteristics of the scatterer, and specific contractions with the directions of incidence, polarization, and observation.

The normalized spherical scattering amplitudes and the scattering cross section are evaluated in Sec. 5. In Sec. 6 we discuss the special cases that correspond to degenerate ellipsoids, such as the sphere, the prolate and the oblate spheroid, the needle and the disc.

The final Sec. 7 includes a discussion of the physical implications of the results and a comparison with the case of the rigid ellipsoid.

2. Formulation of the problem. Let us assume that the space $\mathbf{R}^{3}$ is filled with an isotropic elastic medium characterized by the Lamé constants $\lambda, \underline{\mu}$, and the normalized mass density $\rho=1$. The infinite medium includes the triaxial ellipsoidal cavity

$$
\sum_{i=1}^{3} \frac{x_{i}^{2}}{\alpha_{i}^{2}} \leqslant 1, \quad 0<\alpha_{3}<\alpha_{2}<\alpha_{1}<+\infty,
$$

which represents the scatterer. 
Denote by $V$ the complement of the ellipsoidal cavity and by $S$ its boundary.

The time dependence of the problem is carried through the harmonic factor $\exp \{-i \omega t\}$, which, in the equation for the corresponding spatial oscillation, replaces the explicit time dependence $t$ by the angular frequency $\omega$.

The elastic medium in $V$ is excited by a plane wave $\Phi(\mathbf{r})$, which propagates along the vector $\mathbf{k}$. This incident wave $\Phi(\mathbf{r})$ could be either a plane longitudinal wave ( $P$-wave)

$$
\Phi^{p}(\mathbf{r})=\hat{\mathbf{k}} e^{i k_{p} \hat{\mathbf{k}} \cdot \mathbf{r}}, \quad|\hat{\mathbf{k}}|=1,
$$

or a plane transverse wave ( $S$-wave)

$$
\Phi^{s}(\mathbf{r})=\hat{\mathbf{b}} e^{i k_{\mathbf{s}} \hat{\mathbf{k}} \cdot \mathbf{r}}, \quad|\hat{\mathbf{b}}|=1,
$$

where $\hat{\mathbf{k}}=\left(i_{1}, i_{2}, i_{3}\right), \hat{\mathbf{b}}=\left(b_{1}, b_{2}, b_{3}\right)$, and $\hat{\mathbf{k}} \cdot \hat{\mathbf{b}}=0$. The wave numbers $k_{p}$ and $k_{s}$, which describe the spatial density of the longitudinal and transverse oscillations, respectively, are connected with the corresponding wavelengths $\lambda_{p}$ and $\lambda_{s}$ by

$$
k_{p}=\frac{2 \pi}{\lambda_{p}}, \quad k_{s}=\frac{2 \pi}{\lambda_{s}} .
$$

The ratio of the angular frequency to the corresponding wave number provides the phase velocities $c_{p}$ and $c_{s}$ for the $P$ and $S$ waves, respectively, as

$$
c_{p}^{2}=\frac{\omega^{2}}{k_{p}^{2}}=\lambda+2 \underline{\mu}, \quad c_{s}^{2}=\frac{\omega^{2}}{k_{s}^{2}}=\underline{\mu} .
$$

As a result of the interaction between the incident wave $\Phi$ and the boundary $S$ of the discontinuity in the medium, a scattered wave $\mathbf{u}(\mathbf{r})$ appears in $V$ which satisfies the equation of steady-state elastic oscillations

$$
\underline{\mu} \Delta \mathbf{u}+(\lambda+\underline{\mu}) \nabla \nabla \cdot \mathbf{u}+\omega^{2} \mathbf{u}=\mathbf{0}, \quad \text { in } V .
$$

Due to the linearity of our scattering problem the same equation (6) also describes the total (incident) and scattered field

$$
\Psi(\mathbf{r})=\Phi(\mathbf{r})+\mathbf{u}(\mathbf{r}), \quad \mathbf{r} \in V .
$$

The total field $\Psi$ should satisfy the boundary condition

$$
T \Psi(\mathbf{r})=\mathbf{0}, \quad \mathbf{r} \in S,
$$

where $T$ is the surface traction operator given by

$$
T=2 \underline{\mu} \hat{\mathbf{n}} \cdot \nabla+\lambda \hat{\mathbf{n}} \operatorname{div}+\underline{\mu} \hat{\mathbf{n}} \times \operatorname{rot},
$$

and $\hat{\mathbf{n}}$ is the outward unit normal on $S$.

By Poisson's decomposition [15]

where

$$
\mathbf{u}(\mathbf{r})=\mathbf{u}^{p}(\mathbf{r})+\mathbf{u}^{s}(\mathbf{r}), \quad \mathbf{r} \in V,
$$

$$
\nabla \times \mathbf{u}^{p}=\mathbf{0}, \quad \nabla \cdot \mathbf{u}^{s}=0,
$$

while the longitudinal part $\mathbf{u}^{p}$ and the transverse part $\mathbf{u}^{s}$ satisfy the Kupradze radiation conditions [11]

$$
\begin{aligned}
\lim _{r \rightarrow+\infty} \mathbf{u}^{p}(\mathbf{r})=\mathbf{0}, & \lim _{r \rightarrow+\infty}\left[\partial_{r} \mathbf{u}^{p}(\mathbf{r})-i k_{p} \mathbf{u}^{p}(\mathbf{r})\right] r=\mathbf{0}, \\
\lim _{r \rightarrow+\infty} \mathbf{u}^{s}(\mathbf{r})=\mathbf{0}, & \lim _{r \rightarrow+\infty}\left[\partial_{r} \mathbf{u}^{s}(\mathbf{r})-i k_{s} \mathbf{u}^{s}(\mathbf{r})\right] r=\mathbf{0},
\end{aligned}
$$


where the convergence is uniform over all possible directions and $\partial_{r}$ represents radial differentiation.

In [7] we indicated that the total field $\Psi$ which satisfies the prescribed scattering problem has the following integral representation:

$$
\Psi(\mathbf{r})=\Phi(\mathbf{r})+\frac{1}{4 \pi} \int_{S} \Psi\left(\mathbf{r}^{\prime}\right) \cdot T_{\mathbf{r}} \tilde{\Gamma}\left(\mathbf{r}, \mathbf{r}^{\prime}\right) d s\left(\mathbf{r}^{\prime}\right), \quad \mathbf{r} \in V .
$$

The dyadic $\tilde{\Gamma}\left(\mathbf{r}, \mathbf{r}^{\prime}\right)$ describes the fundamental solution for equation (6), i.e., $\tilde{\Gamma}$ satisfies the dyadic equation

$$
\left[\left(c_{s}^{2} \Delta_{\mathbf{r}^{\prime}}+\omega^{2}\right) \tilde{I}+\left(c_{p}^{2}-c_{s}^{2}\right) \nabla_{\mathbf{r}^{\prime}} \otimes \nabla_{\mathbf{r}^{\prime}}\right] \cdot \tilde{\Gamma}\left(\mathbf{r}, \mathbf{r}^{\prime}\right)=-4 \pi \delta\left(\mathbf{r}-\mathbf{r}^{\prime}\right) \tilde{I}
$$

where $\delta$ is the point-evaluation (Dirac) measure at $\mathbf{r}$ and $\tilde{I}=\hat{\mathbf{x}}_{1} \otimes \hat{\mathbf{x}}_{1}+\hat{\mathbf{x}}_{2} \otimes \hat{\mathbf{x}}_{2}+\hat{\mathbf{x}}_{3} \otimes$ $\hat{\mathbf{x}}_{3}$ is the identity dyadic. The fundamental dyadic $\tilde{\Gamma}$ has the following analytic expression:

$$
\tilde{\Gamma}\left(\mathbf{r}, \mathbf{r}^{\prime}\right)=\frac{k_{s}^{2}}{\omega^{2}} \frac{e^{i k_{s}\left|\mathbf{r}-\mathbf{r}^{\prime}\right|}}{\left|\mathbf{r}-\mathbf{r}^{\prime}\right|} \tilde{I}-\frac{1}{\omega^{2}} \nabla \otimes \nabla\left(\frac{e^{i k_{p}\left|\mathbf{r}-\mathbf{r}^{\prime}\right|}-e^{i k_{s}\left|\mathbf{r}-\mathbf{r}^{\prime}\right|}}{\left|\mathbf{r}-\mathbf{r}^{\prime}\right|}\right),
$$

which can be separated into its $P$ and $S$ parts as

$$
\tilde{\Gamma}\left(\mathbf{r}, \mathbf{r}^{\prime}\right)=\tilde{\Gamma}^{p}\left(\mathbf{r}, \mathbf{r}^{\prime}\right)+\tilde{\Gamma}^{s}\left(\mathbf{r}, \mathbf{r}^{\prime}\right),
$$

where

$$
\begin{aligned}
\tilde{\Gamma}^{p}\left(\mathbf{r}, \mathbf{r}^{\prime}\right)= & \frac{e^{i k_{p}\left|\mathbf{r}-\mathbf{r}^{\prime}\right|}}{\omega^{2}\left|\mathbf{r}-\mathbf{r}^{\prime}\right|^{3}}\left[k_{p}^{2}\left(\mathbf{r}-\mathbf{r}^{\prime}\right) \otimes\left(\mathbf{r}-\mathbf{r}^{\prime}\right)\right. \\
& \left.+\left(1-i k_{p}\left|\mathbf{r}-\mathbf{r}^{\prime}\right|\right)\left(\tilde{I}-3 \frac{\left(\mathbf{r}-\mathbf{r}^{\prime}\right) \otimes\left(\mathbf{r}-\mathbf{r}^{\prime}\right)}{\left|\mathbf{r}-\mathbf{r}^{\prime}\right|^{2}}\right)\right]
\end{aligned}
$$

and

$$
\begin{aligned}
\tilde{\Gamma}^{s}\left(\mathbf{r}, \mathbf{r}^{\prime}\right)= & -\frac{e^{i k_{s}\left|\mathbf{r}-\mathbf{r}^{\prime}\right|}}{\omega^{2}\left|\mathbf{r}-\mathbf{r}^{\prime}\right|^{3}}\left[k_{s}^{2}\left(\mathbf{r}-\mathbf{r}^{\prime}\right) \otimes\left(\mathbf{r}-\mathbf{r}^{\prime}\right)\right. \\
& \left.+\left(1-i k_{s}\left|\mathbf{r}-\mathbf{r}^{\prime}\right|\right)\left(\tilde{I}-3 \frac{\left(\mathbf{r}-\mathbf{r}^{\prime}\right) \otimes\left(\mathbf{r}-\mathbf{r}^{\prime}\right)}{\left|\mathbf{r}-\mathbf{r}^{\prime}\right|^{2}}\right)\right]+\frac{k_{s}^{2}}{\omega^{2}} \frac{e^{i k_{s}\left|\mathbf{r}-\mathbf{r}^{\prime}\right|}}{\left|\mathbf{r}-\mathbf{r}^{\prime}\right|} \tilde{I}
\end{aligned}
$$

In what follows the double contraction is defined as

$$
(\mathbf{a} \otimes \mathbf{b}):(\mathbf{c} \otimes \mathbf{d})=(\mathbf{a} \cdot \mathbf{d})(\mathbf{b} \cdot \mathbf{c}) \text {. }
$$

It is also proved in [7] that the normalized spherical scattering amplitudes for the case of a cavity are given by

$$
\begin{aligned}
& g_{r}(\hat{\mathbf{r}}, \hat{\mathbf{k}})=k_{p}^{2} \tilde{H}_{p}:\left[\frac{\lambda}{\lambda+2 \underline{\mu}} \tilde{I}+\frac{2 \underline{\mu}}{\lambda+2 \underline{\mu}} \hat{\mathbf{r}} \otimes \hat{\mathbf{r}}\right] \\
& g_{\theta}(\hat{\mathbf{r}}, \hat{\mathbf{k}})=k_{s}^{2}\left[2 \tilde{H}_{s}: \hat{\mathbf{r}} \otimes \hat{\boldsymbol{\theta}}+\left(\mathbf{h}_{s} \cdot \hat{\varphi}\right)\right] \\
& g_{\varphi}(\hat{\mathbf{r}}, \hat{\mathbf{k}})=k_{s}^{2}\left[2 \tilde{H}_{s}: \hat{\mathbf{r}} \otimes \hat{\varphi}-\left(\mathbf{h}_{s} \cdot \hat{\boldsymbol{\theta}}\right)\right],
\end{aligned}
$$


where

$$
\begin{aligned}
& \tilde{H}_{p}=\frac{1}{4 \pi} \int_{S} \Psi\left(\mathbf{r}^{\prime}\right) \otimes \hat{\mathbf{n}}^{\prime} e^{-i k_{p} \hat{\mathbf{r}} \cdot \mathbf{r}^{\prime}} d s\left(\mathbf{r}^{\prime}\right), \\
& \tilde{H}_{s}=\frac{1}{4 \pi} \int_{S} \Psi\left(\mathbf{r}^{\prime}\right) \otimes \hat{\mathbf{n}}^{\prime} e^{-i k_{s} \hat{\mathbf{r}} \cdot \mathbf{r}^{\prime}} d s\left(\mathbf{r}^{\prime}\right), \\
& \mathbf{h}_{s}=\frac{1}{4 \pi} \int_{S} \Psi\left(\mathbf{r}^{\prime}\right) \times \hat{\mathbf{n}}^{\prime} e^{-i k_{s} \hat{\mathbf{r}} \cdot \mathbf{r}^{\prime}} d s\left(\mathbf{r}^{\prime}\right),
\end{aligned}
$$

the vector $\mathbf{h}_{s}$ being the vector invariant of the dyadic $\tilde{H}_{s}$.

Perhaps the most important quantity in the classical theory of scattering is the scattering cross section. The scattering cross section is defined as the ratio of the time average rate at which energy is scattered by the body to the corresponding time average rate at which the energy of the incident wave crosses a unit area normal to the direction of propagation. It is obvious from the definition that the scattering cross section is a global measure of the disturbance to the incident wave caused by the existence of the scatterer.

If we denote by $\sigma^{p}$ the scattering cross section corresponding to $P$-incidence and by $\sigma^{s}$ the one corresponding to $S$-incidence, then, as shown in [7], we obtain

$$
\begin{gathered}
\boldsymbol{\sigma}^{p}=k_{p} \int_{|\hat{\mathbf{r}}|=1}\left[k_{p}^{-3}\left|g_{r}^{p}\right|^{2}+k_{s}^{-3}\left(\left|g_{\theta}^{p}\right|^{2}+\left|g_{\varphi}^{p}\right|^{2}\right)\right] d \omega(\hat{\mathbf{r}}), \\
\boldsymbol{\sigma}^{s}=k_{s} \int_{|\hat{\mathbf{r}}|=1}\left[k_{p}^{-3}\left|g_{r}^{s}\right|^{2}+k_{s}^{-3}\left(\left|g_{\theta}^{s}\right|^{2}+\left|g_{\varphi}^{s}\right|^{2}\right)\right] d \omega(\hat{\mathbf{r}}),
\end{gathered}
$$

where the superscripts $p$ and $s$ on the $g$ 's indicate whether the scattering amplitudes come from $P$ - or from $S$-incidence.

The above scattering problem was studied in [7] in the low-frequency region, where the wavelength of the incident plane oscillation is much larger than the characteristic dimension of the scatterer, which is the radius of the smallest sphere that circumscribes the scattering region.

In this case a substitution of the low-frequency expansions

$$
\begin{aligned}
& \Psi(\mathbf{r})=\sum_{n=0}^{\infty} \frac{(i \tau k)^{n}}{n !} \Phi_{n}(\mathbf{r}), \quad \mathbf{r} \in V, \\
& \Phi(\mathbf{r})=\sum_{n=0}^{\infty} \pi^{n} \frac{(i \tau k)^{n}}{n !}(\hat{\mathbf{k}} \cdot \mathbf{r})^{n}, \quad \mathbf{r} \in V,
\end{aligned}
$$

and

$$
\tilde{\Gamma}\left(\mathbf{r}, \mathbf{r}^{\prime}\right)=\frac{1}{\underline{\mu}} \sum_{n=0}^{\infty} \frac{(i \tau k)^{n}}{n !} \tilde{\gamma}_{n}\left(\mathbf{r}, \mathbf{r}^{\prime}\right)
$$


where

$$
\begin{aligned}
& \left.\begin{array}{c}
k=k_{s} \\
\tau=\frac{k_{p}}{k_{s}}=\frac{c_{s}}{c_{p}}=\sqrt{\frac{\underline{\mu}}{\lambda+2 \underline{\mu}}}
\end{array}\right\}, \\
& \pi^{n}=\left\{\begin{array}{lll}
\hat{\mathbf{k}}, & \text { when } & \boldsymbol{\Phi}=\boldsymbol{\Phi}^{p}, \\
\frac{\hat{\mathbf{b}}}{\tau^{n}}, & \text { when } & \boldsymbol{\Phi}=\boldsymbol{\Phi}^{s},
\end{array} \quad n=0,1,2, \ldots,\right.
\end{aligned}
$$

and$$
\tilde{\gamma}_{n}\left(\mathbf{r}, \mathbf{r}^{\prime}\right)=\frac{\left|\mathbf{r}-\mathbf{r}^{\prime}\right|^{n-1}}{\tau^{n}}\left[\left(1+\frac{\tau^{n+2}-1}{n+2}\right) \tilde{I}+(n-1) \frac{\tau^{n+2}-1}{n+2} \frac{\left(\mathbf{r}-\mathbf{r}^{\prime}\right) \otimes\left(\mathbf{r}-\mathbf{r}^{\prime}\right)}{\left|\mathbf{r}-\mathbf{r}^{\prime}\right|^{2}}\right],
$$

$$
n=0,1,2, \ldots,
$$

into all the fundamental equations of our scattering problem reduces it to a sequence of potential problems which can be solved iteratively. In fact, it is proved in [7] that the coefficient $\boldsymbol{\Phi}_{n}$ of the expansion (28) is given by

$$
\Phi_{n}(\mathbf{r})=\mathbf{P}_{n}(\mathbf{r})+\mathbf{U}_{n}(\mathbf{r}),
$$

where for every $n=0,1,2, \ldots$,

$$
\mathbf{P}_{n}(\mathbf{r})=\pi^{n}(\hat{\mathbf{k}} \cdot \mathbf{r})^{n}+\frac{1}{4 \pi \underline{\mu}} \sum_{\rho=0}^{n-1}\left(\begin{array}{l}
n \\
\rho
\end{array}\right) \int_{S} \Phi_{\rho}\left(\mathbf{r}^{\prime}\right) \cdot T_{\mathbf{r}^{\prime}} \tilde{\gamma}_{n-\rho}\left(\mathbf{r}, \mathbf{r}^{\prime}\right) d s\left(\mathbf{r}^{\prime}\right)
$$

and $\mathbf{U}_{n}(\mathbf{r})$ satisfies the following exterior boundary value problem of elastostatics:

$$
\begin{gathered}
\tau^{2} \Delta \mathbf{U}_{n}(\mathbf{r})+\left(1-\tau^{2}\right) \nabla \nabla \cdot \mathbf{U}_{n}(\mathbf{r})=\mathbf{0}, \quad \mathbf{r} \in V, \\
T \mathbf{U}_{n}(\mathbf{r})=-T \mathbf{P}_{n}(\mathbf{r}), \quad r \in S, \\
\mathbf{U}_{n}(\mathbf{r})=O\left(\frac{1}{r}\right), \quad r \rightarrow+\infty .
\end{gathered}
$$

The corresponding low-frequency expansions for the normalized spherical scattering amplitudes are [7]:

$$
\begin{aligned}
g_{r}(\hat{\mathbf{r}}, \hat{\mathbf{k}})= & \frac{1}{4 \pi} \sum_{n=1}^{\infty} \frac{(i \tau k)^{n+2}}{n !} \sum_{\rho=0}^{n}\left(\begin{array}{l}
n \\
\rho
\end{array}\right)(-1)^{\rho+1} \\
& \times\left[\left(1-2 \tau^{2}\right) \tilde{I}+2 \tau^{2} \hat{\mathbf{r}} \otimes \hat{\mathbf{r}}\right]: \int_{S} \Phi_{n-\rho}\left(\mathbf{r}^{\prime}\right) \otimes \hat{\mathbf{n}}^{\prime}\left(\hat{\mathbf{r}} \cdot \mathbf{r}^{\prime}\right)^{\rho} d s\left(\mathbf{r}^{\prime}\right), \\
g_{\theta}(\hat{\mathbf{r}}, \hat{\mathbf{k}})= & \frac{1}{4 \pi \tau} \sum_{n=1}^{\infty} \frac{(i \tau k)^{n+2}}{n !} \sum_{\rho=0}^{n}\left(\begin{array}{l}
n \\
\rho
\end{array}\right)\left(-\frac{1}{\tau}\right)^{\rho+1} \\
& \times \int_{S}\left[2 \hat{\mathbf{r}} \otimes \hat{\theta}: \Phi_{n-\rho}\left(\mathbf{r}^{\prime}\right) \otimes \hat{\mathbf{n}}^{\prime}+\hat{\varphi} \cdot \Phi_{n-\rho}\left(r^{\prime}\right) \times \hat{\mathbf{n}}^{\prime}\right]\left(\hat{\mathbf{r}} \cdot \mathbf{r}^{\prime}\right)^{\rho} d s\left(\mathbf{r}^{\prime}\right), \\
g_{\varphi}(\hat{\mathbf{r}}, \hat{\mathbf{k}})= & \frac{1}{4 \pi \tau} \sum_{n=1}^{\infty} \frac{(i \tau k)^{n+2}}{n !} \sum_{\rho=0}^{n}\left(\begin{array}{l}
n \\
\rho
\end{array}\right)\left(-\frac{1}{\tau}\right)^{\rho+1} \\
& \times \int_{S}\left[2 \hat{\mathbf{r}} \otimes \hat{\varphi}: \Phi_{n-\rho}\left(\mathbf{r}^{\prime}\right) \otimes \hat{\mathbf{n}}^{\prime}-\hat{\boldsymbol{\theta}} \cdot \Phi_{n-\rho}\left(\mathbf{r}^{\prime}\right) \times \hat{\mathbf{n}}^{\prime}\right]\left(\hat{\mathbf{r}} \cdot \mathbf{r}^{\prime}\right)^{\rho} d s\left(\mathbf{r}^{\prime}\right) .
\end{aligned}
$$


Substituting (39)-(41) into (26) and (27), corresponding low-frequency expansions for the scattering cross sections are obtained.

In order to solve the exterior boundary value problem (36)-(38) we use the Papkovich representation [13] in terms of harmonic functions given by

$$
\mathbf{U}_{n}(\mathbf{r})=\mathbf{A}^{n}(\mathbf{r})+\frac{\tau^{2}-1}{2} \nabla\left[\mathbf{r} \cdot \mathbf{A}^{n}(\mathbf{r})+B^{n}(\mathbf{r})\right],
$$

where

$$
\begin{aligned}
& \Delta \mathbf{A}^{n}(\mathbf{r})=\mathbf{0}, \\
& \Delta B^{n}(\mathbf{r})=0 .
\end{aligned}
$$

Although the scalar potential $B^{n}$ is not independent of the vector potential $\mathbf{A}^{n}$ [a fact that is assured from the homogeneity of Eq. (36)], it is very important to introduce the fictitious body force, which is represented by $B^{n}$, in order to use an improved version of the technique introduced in [8] and be able to evaluate the coefficients. In the case of a rigid ellipsoid the boundary condition separates into "Cartesian" and "ellipsoidal" parts, while for a cavity, in addition to these, we must also deal with the "ellipsoidal-derivative" part, which comes from the action of the surface traction operator on the displacement field.

The ellipsoidal coordinates $(\rho, \mu, \nu)$ are related to the Cartesian coordinates $\left(x_{1}, x_{2}, x_{3}\right)$ by

$$
\begin{gathered}
x_{1}=\frac{\rho \mu \nu}{h_{2} h_{3}}, \\
x_{2}=\frac{\sqrt{\rho^{2}-h_{3}^{2}} \sqrt{\mu^{2}-h_{3}^{2}} \sqrt{h_{3}^{2}-\nu^{2}}}{h_{1} h_{3}}, \\
x_{3}=\frac{\sqrt{\rho^{2}-h_{2}^{2}} \sqrt{h_{2}^{2}-\mu^{2}} \sqrt{h_{2}^{2}-\nu^{2}}}{h_{1} h_{2}},
\end{gathered}
$$

where

$$
h_{1}^{2}=a_{2}^{2}-a_{3}^{2}, \quad h_{2}^{2}=a_{1}^{2}-a_{3}^{2}, \quad h_{3}^{2}=a_{1}^{2}-a_{2}^{2}
$$

are the squares of the main semifocal distances and

$$
0 \leqslant \nu^{2} \leqslant h_{3}^{2} \leqslant \mu^{2} \leqslant h_{2}^{2} \leqslant \rho^{2}<+\infty .
$$

The region $V$ corresponds to $\rho>a_{1}$ while the surface $S$ is described by $\rho=a_{1}$.

The appropriate eigenfunction expansions for the Papkovich potentials $\mathbf{A}^{n}$ and $B^{n}$ which, through (42), produce the field $\mathbf{U}_{n}$ that satisfies Equation (36) and the asymptotic condition (38) are given by

$$
\begin{aligned}
& \mathbf{A}^{\prime}(\mathbf{r})=\sum_{n=0}^{\infty} \sum_{m=1}^{2 n+1} \mathbf{c}_{n}^{l, m} \mathbb{F}_{n}^{m}(\rho, \mu, \nu), \\
& B^{l}(\mathbf{r})=\sum_{n=0}^{\infty} \sum_{m=1}^{2 n+1} \beta_{n}^{l, m} \mathbb{F}_{n}^{m}(\rho, \mu, \nu),
\end{aligned}
$$


where $\mathbb{F}_{n}^{m}$ are ellipsoidal harmonics of the second kind (see the Appendix). Substituting (45) and (46) into (42), we obtain

$$
\begin{aligned}
\mathbf{U}_{l}(\mathbf{r})= & \frac{1}{2}\left(\tau^{2}+1\right) \sum_{n=0}^{\infty} \sum_{m=1}^{2 n+1} \mathbf{c}_{n}^{l, m} \mathbb{F}_{n}^{m}(\rho, \mu, \nu) \\
& +\frac{1}{2}\left(\tau^{2}-1\right) \sum_{n=0}^{\infty} \sum_{m=0}^{2 n+1}\left(\mathbf{r} \cdot \mathbf{c}_{n}^{l, m}+\beta_{n}^{l, m}\right) \nabla \mathbb{F}_{n}^{m}(\rho, \mu, \nu) .
\end{aligned}
$$

On the surface $\rho=a_{1}$ of the scatterer the action of the surface stress operator $T$ on $\mathbf{U}_{l}$ should be equal to the action of $T$ on $-\mathbf{P}_{l}$, where $\mathbf{P}_{l}$ is given by (35). We observe that $\mathbf{P}_{l}$ is of degree $l$ in the Cartesian components $x_{1}, x_{2}, x_{3}$ of $\mathbf{r}$. Hence, since $T$ includes one differentiation in each term, $T \mathbf{P}_{l}$ is of degree $l-1$ in $x_{i}$ and the same is true for $T \mathbf{U}_{l}$. Therefore, $\mathbf{U}_{l}$ should be of degree $l$ in $x_{i}$, or equivalently $\mathbf{U}_{l}$ should include ellipsoidal harmonics of degree not greater than $l$. This shows the completeness of the finite expansion [8]

$$
\begin{aligned}
& \mathbf{U}_{l}(\mathbf{r})=\frac{1}{2}\left\{\left(\tau^{2}+1\right) \sum_{n=0}^{l} \sum_{m=1}^{2 n+1} \mathbf{c}_{n}^{l, m}(2 n+1) I_{n}^{m}(\rho) \mathbb{E}_{n}^{m}(\rho, \mu, \nu)\right. \\
& +\left(\tau^{2}-1\right) \sum_{n=0}^{l} \sum_{m=1}^{2 n+1}\left(\mathbf{r} \cdot \mathbf{c}_{n}^{l, m}\right)(2 n+1) I_{n}^{m}(\rho) \nabla \mathbb{E}_{n}^{m}(\rho, \mu, \nu) \\
& \left.+\left(\tau^{2}-1\right) \sum_{n=0}^{l+1} \sum_{m=1}^{2 n+1} \beta_{n}^{l, m}(2 n+1) I_{n}^{m}(\rho) \nabla \mathbb{E}_{n}^{m}(\rho, \mu, \nu)\right\} \\
& -\frac{1}{2}\left(\tau^{2}-1\right) \frac{\hat{\mathbf{\rho}}}{\sqrt{\rho^{2}-\mu^{2}} \sqrt{\rho^{2}-\nu^{2}}}\left\{\sum_{n=0}^{l} \sum_{m=1}^{2 n+1}\left(\mathbf{r} \cdot \mathbf{c}_{n}^{l, m}\right)(2 n+1)\left(E_{n}^{m}(\rho)\right)^{-2} \mathbb{E}_{n}^{m}(\rho, \mu, \nu)\right. \\
& \left.+\sum_{n=0}^{l+1} \sum_{m=1}^{2 n+1} \beta_{n}^{l, m}(2 n+1)\left(E_{n}^{m}(\rho)\right)^{-2} \mathbb{E}_{n}^{m}(\rho, \mu, \nu)\right\}
\end{aligned}
$$

where

$$
h_{\rho}=\frac{\sqrt{\rho^{2}-\mu^{2}} \sqrt{\rho^{2}-\nu^{2}}}{\sqrt{\rho^{2}-h_{2}^{2}} \sqrt{\rho^{2}-h_{3}^{2}}}
$$

is the square root of the ellipsoidal metric coefficient that corresponds to the variable $\rho$,

$$
\hat{\boldsymbol{\rho}}=\frac{\rho}{h_{\rho}} \sum_{i=1}^{3} \frac{x_{i}}{\rho^{2}-a_{1}^{2}+a_{i}^{2}} \hat{\mathbf{x}}_{i}
$$

is the unit curvilinear vector relative to the variable $\rho$, and $\hat{\mathbf{x}}_{i}, i=1,2,3$, are the Cartesian base vectors.

3. The zeroth-order field. In order to evaluate the zeroth-order field $\Phi_{0}$ we only need to determine the coefficients $\mathbf{c}_{n}^{0, m}$ and $\beta_{n}^{0, m}$ in the expansion (47) in such a way that

$$
T \Phi_{0}=T\left(\pi^{0}+\mathbf{U}_{0}\right)=T \mathbf{U}_{0}=\mathbf{0}
$$


on the surface $\rho=a_{1}$ of the ellipsoid. It was shown in [8] that the field $\mathbf{U}_{0}$ has the following expansion in terms of surface ellipsoidal harmonics:

$$
\begin{aligned}
\mathbf{U}_{0}(\rho, \mu, \nu)= & \frac{1}{2}\left(\tau^{2}-1\right)\left\{\frac{\tau^{2}+1}{\tau^{2}-1} \mathbf{c}_{0}^{0,1} I_{0}^{1}(\rho)+3 h_{1} h_{2} h_{3} \sum_{m=1}^{3} \frac{\beta_{1}^{0, m}}{h_{m}} I_{1}^{m}(\rho) \hat{\mathbf{x}}_{m}\right\} \\
& -\frac{1}{2}\left(\tau^{2}-1\right) \mathbf{R}^{e}\left\{\beta_{0}^{0,1}+\sum_{m=1}^{3}\left(\frac{c_{0 m}^{0,1} h_{m} E_{1}^{m}(\rho)}{h_{1} h_{2} h_{3}}+\frac{3 \beta_{1}^{0, m}}{E_{1}^{m}(\rho)}\right) E_{1}^{m}(\mu) E_{1}^{m}(\nu)\right\},
\end{aligned}
$$

where

$$
\mathbf{R}^{e}=\left(\rho^{2}-\mu^{2}\right)^{-1 / 2}\left(\rho^{2}-\nu^{2}\right)^{-1 / 2} \hat{\boldsymbol{\rho}} .
$$

The first bracket in (52) is the Cartesian part and the second bracket is the ellipsoidal part of $\mathbf{U}_{0}$. Since

$$
\begin{aligned}
& \hat{\mathbf{n}} \cdot \nabla \mathbf{U}_{0}=\frac{a_{2} a_{3}}{\sqrt{a_{1}^{2}-\mu^{2}} \sqrt{a_{1}^{2}-\nu^{2}}} \frac{\partial}{\partial \rho} \mathbf{U}_{0}, \\
& \nabla \cdot \mathbf{U}_{0}=-\frac{\tau^{2}}{\sqrt{a_{1}^{2}-\mu^{2}} \sqrt{a_{1}^{2}-\nu^{2}}} \hat{\boldsymbol{\rho}} \cdot \mathbf{c}_{0}^{0,1},
\end{aligned}
$$

and

$$
\nabla \times \mathbf{U}_{0}=-\frac{1}{\sqrt{a_{1}^{2}-\mu^{2}} \sqrt{a_{1}^{2}-\nu^{2}}} \hat{\boldsymbol{\rho}} \times \mathbf{c}_{0}^{0,1},
$$

the boundary condition (51) provides the following relation on the surface of the ellipsoid:

$$
\begin{gathered}
\frac{2 \underline{\mu} a_{2} a_{3}}{\sqrt{a_{1}^{2}-\mu^{2}} \sqrt{a_{1}^{2}-\nu^{2}}}\left\{\frac{\tau^{2}+1}{2} \mathbf{c}_{0}^{0,1}\left(-\frac{1}{a_{2} a_{3}}\right)+\frac{3\left(\tau^{2}-1\right)}{2} h_{1} h_{2} h_{3} \sum_{m=1}^{3} \frac{\beta_{1}^{0, m}}{h_{m}}\left(-\frac{1}{a_{m}^{2} a_{2} a_{3}}\right) \hat{\mathbf{x}}_{m}\right\} \\
-\frac{2 \mu a_{2} a_{3}}{\sqrt{a_{1}^{2}-\mu^{2}} \sqrt{a_{1}^{2}-\nu^{2}}} \frac{\tau^{2}-1}{2}\left(\frac{\partial \mathbf{R}^{e}}{\partial \rho}\right)\left\{\beta_{0}^{0,1}+\sum_{m=1}^{3}\left(\frac{c_{0 m}^{0,1} h_{m} a_{m}}{h_{1} h_{2} h_{3}}+\frac{3 \beta_{1}^{0, m}}{a_{m}}\right) E_{1}^{m}(\mu) E_{1}^{m}(\nu)\right\} \\
-\frac{2 \mu a_{2} a_{3}}{\sqrt{a_{1}^{2}-\mu^{2}} \sqrt{a_{1}^{2}-\nu^{2}}} \frac{\tau^{2}-1}{2} \mathbf{R}^{e} \sum_{m=1}^{3}\left(\frac{c_{0 m}^{0,1} h_{m} a_{1}}{h_{1} h_{2} h_{3} a_{m}}-\frac{3 \beta_{1}^{0, m} a_{1}}{a_{m}^{3}}\right) E_{1}^{m}(\mu) E_{1}^{m}(\nu) \\
-\frac{\lambda \tau^{2}}{\sqrt{a_{1}^{2}-\mu^{2}} \sqrt{a_{1}^{2}-\nu^{2}}} \hat{\boldsymbol{\rho}}\left(\hat{\mathbf{\rho}} \cdot \mathbf{c}_{0}^{0,1}\right)-\frac{\underline{\mu}}{\sqrt{a_{1}^{2}-\mu^{2}} \sqrt{a_{1}^{2}-\nu^{2}}} \hat{\boldsymbol{\rho}} \times\left(\hat{\mathbf{\rho}} \times \mathbf{c}_{0}^{0,1}\right)=\mathbf{0} .
\end{gathered}
$$

The above form of the boundary condition involves three types of terms. Terms, such as those in the first bracket, that do not contain the characteristic ellipsoidal vector $\mathbf{R}^{e}$ constitute the Cartesian part of the boundary relation. The ellipsoid part is constituted by all the terms that involve the vector $\mathbf{R}^{e}$. Finally, all the terms that contain the normal derivative $\partial_{\rho} \mathbf{R}^{e}$ form the ellipsoidal-derivative part. Now looking at the boundary condition as consisting of the three parts above and using the dependence of the scalar on the 
vector Papkovich potential, we establish the relation between the $\beta$ and the coefficients by vanishing the ellipsoidal-derivative part independently. This will give us the relations

$$
\left.\begin{array}{l}
\beta_{0}^{0,1}=0, \\
\beta_{1}^{0, m}=-\frac{h_{m} a_{m}^{2}}{3 h_{1} h_{2} h_{3}} c_{0 m}^{0,1}, \quad m=1,2,3 .
\end{array}\right\}
$$

Note that these expressions are the same as the corresponding relations for the rigid ellipsoid [8] [relations (64)] since the vanishing factor that reduces to (58) is connected to the ellipsoidal part in the case of a rigid scatterer, while for the cavity it is connected to the ellipsoidal-derivative part. The additional difficulty that appears in our case of a cavity is due to the fact that, after establishing the connection between the $\beta$ 's and the c's, we are still left with two types of terms and with no more freedom to vanish terms independently. In fact, if we substitute (58) into (57) we obtain

$$
\begin{aligned}
\underline{\mu} \mathbf{c}_{0}^{0,1} & +\left(\lambda \tau^{2}+\underline{\mu}\right) \hat{\boldsymbol{\rho}}\left(\hat{\boldsymbol{\rho}} \cdot \mathbf{c}_{0}^{0,1}\right) \\
& +2 \underline{\mu}\left(\tau^{2}-1\right) a_{1} a_{2} a_{3} \frac{\hat{\boldsymbol{\rho}}}{\sqrt{a_{1}^{2}-\mu^{2}} \sqrt{a_{1}^{2}-\nu^{2}}} \sum_{m=1}^{3} \frac{x_{m}}{a_{m}^{2}} c_{0 m}^{0.1}=\mathbf{0} .
\end{aligned}
$$

In view of the relation

$$
\hat{\boldsymbol{\rho}}=\frac{a_{1} a_{2} a_{3}}{\sqrt{a_{1}^{2}-\mu^{2}} \sqrt{a_{1}^{2}-\nu^{2}}} \sum_{m=1}^{3} \frac{x_{m}}{a_{m}^{2}} \hat{\mathbf{x}}_{m},
$$

the boundary condition (59) assumes the form

$$
\underline{\mu} \mathbf{c}_{0}^{0,1}+\left[\left(\lambda \tau^{2}+\underline{\mu}\right)+2 \underline{\mu}\left(\tau^{2}-1\right)\right] \hat{\rho}\left(\hat{\boldsymbol{\rho}} \cdot \mathbf{c}_{0}^{0,1}\right)=\mathbf{0},
$$

and since $\tau^{2}=\underline{\mu} /(\lambda+2 \underline{\mu}), \underline{\mu}>0,(61)$ reduces to

$$
\mathbf{c}_{0}^{0,1}=\mathbf{0} \text {. }
$$

By virtue of (58) and (62), relation (52) implies

$$
\mathbf{U}_{0}(\rho, \mu, \nu)=\mathbf{0}
$$

and

$$
\Phi_{0}(\mathbf{r})=\pi^{0} .
$$

Therefore, the zeroth-order low-frequency approximation for a longitudinal wave scattered by an ellipsoidal cavity is

$$
\Phi_{0}^{p}(\mathbf{r})=\hat{\mathbf{k}},
$$

while for a transverse wave scattered by an ellipsoidal cavity it is

$$
\Phi_{0}^{s}(\mathbf{r})=\hat{\mathbf{b}} .
$$

4. The first-order field. The first-order field has the form

$$
\Phi_{1}(\mathbf{r})=\pi^{1}(\hat{\mathbf{k}} \cdot \mathbf{r})+\mathbf{U}_{1}(\mathbf{r})
$$


where the general polarization vector $\pi^{1}$ is equal to $\hat{\mathbf{k}}$ for $P$-incidence and to $\tau^{-1} \hat{\mathbf{b}}$ for $S$-incidence. The field $\Phi_{1}(\mathbf{r})$ should satisfy the boundary condition

$$
T \Phi_{1}(\mathbf{r})=\mathbf{0}, \quad \rho=a_{1},
$$

and the asymptotic relation

$$
\Phi_{1}(\mathbf{r})=\pi^{1}(\hat{\mathbf{k}} \cdot \mathbf{r})+O\left(\frac{1}{r}\right), \quad r \rightarrow+\infty .
$$

Condition (69) is automatically satisfied since we assumed that $\mathbf{U}_{1}(\mathbf{r})$ has the particular expansion given by (48) for $l=1$. The finite expansion (48) involves Cartesian as well as ellipsoidal expressions for the observation variables. Therefore, our objective is to express all the observation variables is terms of surface ellipsoidal harmonics and then use the orthogonality of these harmonics to evaluate the coefficients.

In fact, we have

$$
\pi^{1}(\hat{\mathbf{k}} \cdot \mathbf{r})=\frac{\pi^{1}}{h_{1} h_{2} h_{3}} \sum_{m=1}^{3} i_{m} h_{m} \mathbb{E}_{1}^{m}(\rho, \mu, \nu),
$$

and using the formulae in the Appendix, $\mathbf{U}_{1}(\mathbf{r})$ assumes the form

$$
\mathbf{U}_{1}(\mathbf{r})=\mathbf{U}_{1}^{\text {cart }}+\mathbf{R}^{e} U_{1}^{\text {ellip }}(\mathbf{r}),
$$

where

$$
\begin{aligned}
& \mathbf{U}_{1}^{\text {cart }}= \frac{\tau^{2}-1}{2}\left\{\left[\frac{\tau^{2}+1}{\tau^{2}-1} \mathbf{c}_{0}^{1,1} I_{0}^{1}(\rho)+3 h_{1} h_{2} h_{3} \sum_{k=1}^{3} \beta_{1}^{1, k} I_{1}^{k}(\rho) \frac{\hat{\mathbf{x}}_{k}}{h_{k}}\right]\right. \\
&+ \sum_{n=1}^{3}\left[3 \frac{\tau^{2}+1}{\tau^{2}-1} \mathbf{c}_{1}^{1, n} I_{1}^{n}(\rho)+3 \sum_{k=1}^{3} \frac{h_{n}}{h_{k}} c_{1 n}^{1, k} I_{1}^{k}(\rho) \hat{\mathbf{x}}_{k}\right. \\
&+ 10 \beta_{2}^{1,1} I_{2}^{1}(\rho) \frac{h_{n}}{h_{1} h_{2} h_{3}} \frac{\left(\Lambda-a_{1}^{2}\right)\left(\Lambda-a_{2}^{2}\right)\left(\Lambda-a_{3}^{2}\right)}{\Lambda-a_{n}^{2}} \hat{\mathbf{x}}_{n} \\
&\left.+10 \beta_{2}^{1,2} I_{2}^{2}(\rho) \frac{h_{n}}{h_{1} h_{2} h_{3}} \frac{\left(\Lambda^{\prime}-a_{1}^{2}\right)\left(\Lambda^{\prime}-a_{2}^{2}\right)\left(\Lambda^{\prime}-a_{3}^{2}\right)}{\Lambda^{\prime}-a_{n}^{2}} \hat{\mathbf{x}}_{n}\right] \mathbb{E}_{1}^{n}(\rho, \mu, \nu) \\
&+5 h_{1} h_{2} h_{3}\left(\beta_{2}^{1,3} I_{2}^{3}(\rho) \frac{\hat{\mathbf{x}}_{2}}{h_{2}}+\beta_{2}^{1,4} I_{2}^{4}(\rho) \frac{\hat{\mathbf{x}}_{3}}{h_{3}}\right) \mathbb{E}_{1}^{1}(\rho, \mu, \nu) \\
&+5 h_{1} h_{2} h_{3}\left(\beta_{2}^{1,3} I_{2}^{3}(\rho) \frac{\hat{\mathbf{x}}_{1}}{h_{1}}+\beta_{2}^{1,5} I_{2}^{5}(\rho) \frac{\hat{\mathbf{x}}_{3}}{h_{3}}\right) \mathbb{E}_{1}^{2}(\rho, \mu, \nu) \\
&\left.+5 h_{1} h_{2} h_{3}\left(\beta_{2}^{1,4} I_{2}^{4}(\rho) \frac{\hat{\mathbf{x}}_{1}}{h_{1}}+\beta_{2}^{1,5} I_{2}^{5}(\rho) \frac{\hat{\mathbf{x}}_{2}}{h_{2}}\right) \mathbb{E}_{1}^{3}(\rho, \mu, \nu)\right\},
\end{aligned}
$$


the ellipsoidal vector $\mathbf{R}^{e}$ is given by (53), and

$$
\begin{aligned}
U_{1}^{\text {ellip }}(\mathbf{r})= & -\frac{\tau^{2}-1}{2}\left\{\left[h_{1} h_{2} h_{3} \sum_{k=1}^{3} \frac{1}{h_{k}} c_{1 k}^{1, k}+\beta_{0}^{1,1}\right]\right. \\
& +\sum_{n=1}^{3}\left[\frac{h_{n}}{h_{1} h_{2} h_{3}} c_{0 n}^{1,1}+3 \beta_{1}^{1, n} \frac{1}{\left(E_{1}^{n}(\rho)\right)^{2}}\right] \mathbb{E}_{1}^{n}(\rho, \mu, \nu) \\
& +\left[\frac{5 \beta_{2}^{1,1}}{E_{2}^{1}(\rho)}-\frac{3}{h_{1} h_{2} h_{3}\left(\Lambda-\Lambda^{\prime}\right)}\left(c_{11}^{1,1} h_{1}\left(\Lambda^{\prime}-a_{1}^{2}\right)\right.\right. \\
& \left.\left.-c_{12}^{1,2} h_{2}\left(\Lambda^{\prime}-a_{2}^{2}\right)+c_{13}^{1,3} h_{3}\left(\Lambda^{\prime}-a_{3}^{2}\right)\right)\right] E_{2}^{1}(\mu) E_{2}^{1}(\nu) \\
& +\left[\frac{5 \beta_{2}^{1,2}}{E_{2}^{2}(\rho)}+\frac{3}{h_{1} h_{2} h_{3}\left(\Lambda-\Lambda^{\prime}\right)}\left(c_{11}^{1,1} h_{1}\left(\Lambda-a_{1}^{2}\right)\right.\right. \\
& \left.\left.-c_{12}^{1,2} h_{2}\left(\Lambda-a_{2}^{2}\right)+c_{13}^{1,3} h_{3}\left(\Lambda-a_{3}^{2}\right)\right)\right] E_{2}^{2}(\mu) E_{2}^{2}(\mu) \\
+ & {\left[\frac{5 \beta_{2}^{1,3}}{\left(E_{2}^{3}(\rho)\right)^{2}}+\frac{3}{h_{3}}\left(\frac{c_{12}^{1,1}}{h_{1}\left(E_{1}^{1}(\rho)\right)^{2}}+\frac{c_{11}^{1,2}}{h_{2}\left(E_{1}^{2}(\rho)\right)^{2}}\right)\right] \mathbb{E}_{2}^{3}(\rho, \mu, \nu) } \\
+ & {\left[\frac{5 \beta_{2}^{1,4}}{\left(E_{2}^{4}(\rho)\right)^{2}}+\frac{3}{h_{2}}\left(\frac{c_{13}^{1,1}}{h_{1}\left(E_{1}^{1}(\rho)\right)^{2}}+\frac{c_{11}^{1,3}}{h_{3}\left(E_{1}^{3}(\rho)\right)^{2}}\right)\right] \mathbb{E}_{2}^{4}(\rho, \mu, \nu) } \\
+ & {\left[\frac{5 \beta_{2}^{1,5}}{\left(E_{2}^{5}(\rho)\right)^{2}}+\frac{3}{h_{1}}\left(\frac{c_{12}^{1,3}}{h_{3}\left(E_{1}^{3}(\rho)\right)^{2}}+\frac{c_{13}^{1,2}}{h_{2}\left(E_{1}^{2}(\rho)\right)^{2}}\right) \mathbb{E}_{2}^{5}(\rho, \mu, \nu)\right\} }
\end{aligned}
$$

The surface traction operator $T$ has the following expression in ellipsoidal coordinates:

$$
T=2 \underline{\mu} \frac{a_{2} a_{3}}{\sqrt{a_{1}^{2}-\mu^{2}} \sqrt{a_{1}^{2}-\nu}} \frac{\partial}{\partial \rho}+\lambda \hat{\boldsymbol{\rho}} \operatorname{div}+\underline{\mu} \hat{\hat{\rho}} \times \operatorname{rot} .
$$

Applying $T$, as given by (74), in the expression

$$
\Phi_{1}(\mathbf{r})=\pi^{1}(\hat{\mathbf{k}} \cdot \mathbf{r})+\mathbf{U}_{1}^{\text {cart }}(\mathbf{r})+\mathbf{R}^{e} U_{1}^{\text {ellip }}(\mathbf{r}),
$$

and using the formulae

$$
\begin{aligned}
& \operatorname{div} \mathbf{U}_{1}(\mathbf{r})=\tau^{2} \operatorname{div} \mathbf{A}^{1}(\mathbf{r}), \\
& \operatorname{rot} \mathbf{U}_{1}(\mathbf{r})=\operatorname{rot} \mathbf{A}^{1}(\mathbf{r}),
\end{aligned}
$$


which can be proved from expression (42) for $n=1$, we obtain the boundary condition in the form

$$
\begin{aligned}
& {\left[\underline{\mu} \pi^{1}(\hat{\boldsymbol{\rho}} \cdot \hat{\mathbf{k}})+\lambda \hat{\boldsymbol{\rho}}\left(\boldsymbol{\pi}^{1} \cdot \hat{\mathbf{k}}\right)+\underline{\mu} \hat{\mathbf{k}}\left(\hat{\boldsymbol{\rho}} \cdot \pi^{1}\right)\right] } \\
&+2 \underline{\mu} \frac{a_{2} a_{3}}{\sqrt{a_{1}^{2}-\mu^{2}} \sqrt{a_{1}^{2}-\nu^{2}}}\left[\frac{\partial}{\partial \rho} \mathbf{U}_{1}^{\text {cart }}(\mathbf{r})+\left(\frac{\partial}{\partial \rho} \mathbf{R}^{e}\right) U_{1}^{\text {ellip }}(\mathbf{r})\right. \\
&\left.+\mathbf{R}^{e}\left(\frac{\partial}{\partial \rho} U_{1}^{\text {ellip }}(\mathbf{r})\right)\right]+\lambda \tau^{2} \hat{\boldsymbol{\rho}} \nabla \cdot \mathbf{A}^{1}+\underline{\mu} \hat{\boldsymbol{\rho}} \times\left(\nabla \times \mathbf{A}^{1}\right)=\mathbf{0},
\end{aligned}
$$

where everything is expressed at $\rho=a_{1}$.

The last two terms in (78) can be written as a sum of a Cartesian plus an ellipsoidal part as follows:

$$
\begin{aligned}
\lambda \tau^{2} \hat{\boldsymbol{\rho}} \nabla \cdot \mathbf{A}^{1}+ & \underline{\mu} \hat{\boldsymbol{\rho}} \times\left(\nabla \times \mathbf{A}^{1}\right) \\
= & \left\{\frac{\underline{\mu}}{\sqrt{a_{1}^{2}-\mu^{2}} \sqrt{a_{1}^{2}-\nu^{2}}}\left(\mathbf{c}_{0}^{1,1}+3 \sum_{m=1}^{3} \frac{\mathbf{c}_{1}^{1, m}}{a_{m}} E_{1}^{m}(\mu) E_{1}^{m}(\nu)\right)\right. \\
& \left.+3 h_{1} h_{2} h_{3} \sum_{m=1}^{3} \frac{I_{1}^{m}}{h_{m}}\left[\lambda \tau^{2} c_{1 m}^{1, m} \hat{\boldsymbol{\rho}}+\underline{\mu}\left(\hat{\boldsymbol{\rho}} \cdot \mathbf{c}_{1}^{1, m}\right) \hat{\mathbf{x}}_{m}-\underline{\mu}\left(\hat{\boldsymbol{\rho}} \cdot \hat{\mathbf{x}}_{m}\right) \mathbf{c}_{1}^{1, m}\right]\right\} \\
& +\mathbf{R}^{\mathbf{e}}\left\{2 \underline{\mu}\left(\tau^{2}-1\right)\left(\hat{\boldsymbol{\rho}} \cdot \mathbf{c}_{0}^{1,1}\right)+6 \underline{\mu}\left(\tau^{2}-1\right) \sum_{m=1}^{3} \frac{\left(\hat{\boldsymbol{\rho}} \cdot \mathbf{c}_{1}^{1, m}\right)}{a_{m}} E_{1}^{m}(\mu) E_{1}^{m}(\nu)\right\} .
\end{aligned}
$$

Vanishing independently the ellipsoidal-derivative term

$$
2 \underline{\mu} \frac{a_{2} a_{3}}{\sqrt{a_{1}^{2}-\mu^{2}} \sqrt{a_{1}^{2}-\mu^{2}}}\left(\frac{\partial}{\partial \rho} \mathbf{R}^{e}\right) U_{1}^{\text {ellip }}(\mathbf{r})
$$

in (78) we obtain

$$
\left.U_{1}^{\text {ellip }}(\mathbf{r})\right|_{\rho=a_{1}}=0,
$$

which finally provides the following relations between the $\beta$ and the $\mathbf{c}$ constants:

$$
\begin{aligned}
& \beta_{0}^{1,1}=-h_{1} h_{2} h_{3} \sum_{k=1}^{3} \frac{1}{h_{k}} c_{1 k}^{1, k} \\
& \beta_{1}^{1, k}=-\frac{h_{k} a_{k}^{2}}{3 h_{1} h_{2} h_{3}} c_{0 k}^{1,1}, \quad k=1,2,3 \\
& \beta_{2}^{1,1}=\frac{3 \Lambda}{5 h_{1} h_{2} h_{3}\left(\Lambda-\Lambda^{\prime}\right)}\left[c_{11}^{1,1} h_{1}\left(\Lambda^{\prime}-a_{1}^{2}\right)-c_{12}^{1,2} h_{2}\left(\Lambda^{\prime}-a_{2}^{2}\right)+c_{13}^{1,3} h_{3}\left(\Lambda^{\prime}-a_{3}^{2}\right)\right]
\end{aligned}
$$




$$
\begin{aligned}
& \beta_{2}^{1,2}=-\frac{3 \Lambda^{\prime}}{5 h_{1} h_{2} h_{3}\left(\Lambda-\Lambda^{\prime}\right)}\left[c_{11}^{1,1} h_{1}\left(\Lambda-a_{1}^{2}\right)-c_{12}^{1,2} h_{2}\left(\Lambda-a_{2}^{2}\right)+c_{13}^{1,3} h_{3}\left(\Lambda-a_{3}^{2}\right)\right] \\
& \beta_{2}^{1,3}=-\frac{3}{5 h_{3}}\left(\frac{a_{1}^{2}}{h_{2}} c_{11}^{1,2}+\frac{a_{2}^{2}}{h_{1}} c_{12}^{1,1}\right) \\
& \beta_{2}^{1,4}=-\frac{3}{5 h_{2}}\left(\frac{a_{1}^{2}}{h_{3}} c_{11}^{1,3}+\frac{a_{3}^{2}}{h_{1}} c_{13}^{1,1}\right) \\
& \beta_{2}^{1,5}=-\frac{3}{5 h_{1}}\left(\frac{a_{2}^{2}}{h_{3}} c_{12}^{1,3}+\frac{a_{3}^{2}}{h_{2}} c_{13}^{1,2}\right)
\end{aligned}
$$

The boundary condition (78), in view of (79) and (80), assumes the form

$$
\begin{aligned}
& \left\{\underline{\mu} \pi^{1}(\hat{\boldsymbol{\rho}} \cdot \hat{\mathbf{k}})+\lambda \hat{\boldsymbol{\rho}}\left(\pi^{1} \cdot \hat{\mathbf{k}}\right)+\underline{\mu} \hat{\mathbf{k}}\left(\hat{\mathbf{\rho}} \cdot \pi^{1}\right)\right. \\
& +\frac{\underline{\mu}}{\sqrt{a_{1}^{2}-\mu^{2}} \sqrt{a_{1}^{2}-\nu^{2}}}\left(\mathbf{c}_{0}^{1.1}+3 \sum_{m=1}^{3} \frac{\mathbf{c}_{1}^{1 . m}}{a_{m}} E_{1}^{m}(\mu) E_{1}^{m}(\nu)\right) \\
& +3 h_{1} h_{2} h_{3} \sum_{m=1}^{3} \frac{I_{1}^{m}}{h_{m}}\left[\lambda \tau^{2} c_{1 m}^{1, m} \hat{\boldsymbol{\rho}}+\underline{\mu}\left(\hat{\boldsymbol{\rho}} \cdot \mathbf{c}_{1}^{1, m}\right) \hat{\mathbf{x}}_{m}-\underline{\mu}\left(\hat{\boldsymbol{\rho}} \cdot \hat{\mathbf{x}}_{m}\right) \mathbf{c}_{1}^{1, m}\right] \\
& \left.+2 \underline{\mu} \frac{a_{2} a_{3}}{\sqrt{a_{1}^{2}-\mu^{2}} \sqrt{a_{1}^{2}-\nu^{2}}}\left(\frac{\partial}{\partial \rho} \mathbf{U}_{1}^{\text {cart }}(\mathbf{r})\right)\right\} \\
& +\mathbf{R}^{e}\left\{2 \underline{\mu} \frac{a_{2} a_{3}}{\sqrt{a_{1}^{2}-\mu^{2}} \sqrt{a_{1}^{2}-\nu^{2}}}\left(\frac{\partial}{\partial \rho} U_{1}^{\text {ellip }}(\mathbf{r})\right)+2 \underline{\mu}\left(\tau^{2}-1\right)\left(\hat{\mathbf{\rho}} \cdot \hat{\mathbf{c}}_{0}^{1,1}\right)\right. \\
& \left.+6 \underline{\mu}\left(\tau^{2}-1\right) \sum_{m=1}^{3} \frac{\left(\hat{\mathbf{\rho}} \cdot \mathbf{c}_{1}^{1, m}\right)}{a_{m}} E_{1}^{m}(\mu) E_{1}^{m}(\nu)\right\}=\mathbf{0},
\end{aligned}
$$

where everything is evaluated at $\rho=a_{1}$.

In the second expression in braces in (88), which represents the ellipsoidal part $\mathbf{R}^{e}\{\cdots\}$, we perform the following operations:

(i) we express the last term inside the braces in terms of surface ellipsoidal harmonics;

(ii) we operate with $\partial / \partial \rho$ on $U_{i}^{\text {ellip }}(\mathbf{r})$ as given by (73);

(iii) we substitute the $\beta$ 's in terms of the c's via formulae (81) to (87);

(iv) we evaluate all the expressions at $\rho=a_{1}$.

Then, after long and tedious calculations and the use of expressions (50), (53), and (A.5)-(A.13) of the appendix, the relation

$$
\Lambda \Lambda^{\prime}-\frac{\Lambda^{\prime} E_{2}^{1}(\mu) E_{2}^{1}(\nu)-\Lambda E_{2}^{2}(\mu) E_{2}^{2}(\nu)}{\Lambda-\Lambda^{\prime}}=\left(a_{1}^{2}-\mu^{2}\right)\left(a_{1}^{2}-\nu^{2}\right),
$$


as well as many other well-known relations connecting the various ellipsoidal characteristics we obtain for the ellipsoidal part of (88):

$$
R^{e}\{\cdots\}=2 \underline{\mu} \frac{a_{1} a_{2} a_{3} h_{1} h_{2} h_{3}}{\Lambda \Lambda^{\prime}}\left(\tau^{2}-1\right) \hat{\rho} \sum_{m=1}^{3} \frac{c_{1 m}^{1, m}}{h_{m} a_{m}^{2}},
$$

which is actually a Cartesian-type term and can be incorporated with the first expression in braces in Equation (88).

Using (90), the expression

$$
\left.\hat{\boldsymbol{\rho}}\right|_{\rho=a_{1}}=\frac{a_{1} a_{2} a_{3}}{h_{1} h_{2} h_{3}} \frac{1}{\sqrt{a_{1}^{2}-\mu^{2}} \sqrt{a_{1}^{2}-\nu^{2}}} \sum_{m=1}^{3} \frac{h_{m}}{a_{m}} E_{1}^{m}(\mu) E_{1}^{m}(\nu) \hat{\mathbf{x}}_{m}
$$

for the unit outward normal on $S$, and relations (81)-(87), the boundary condition can be written as

$$
\begin{aligned}
& -\frac{\underline{\mu}}{\sqrt{a_{1}^{2}-\mu^{2}} \sqrt{a_{1}^{2}-\nu^{2}}} \mathbf{c}_{0}^{1,1}+\frac{\underline{\mu}}{\sqrt{a_{1}^{2}-\mu^{2}} \sqrt{a_{1}^{2}-\nu^{2}}} \sum_{m=1}^{3}\left\{\frac{3}{a_{m}} \mathbf{c}_{1}^{1, m}\right. \\
& +\frac{a_{1} a_{2} a_{3}}{\underline{\mu} h_{1} h_{2} h_{3}} \frac{h_{m}}{a_{m}}\left[\underline{\mu}\left(\pi_{m}^{1} \hat{\mathbf{k}}+i_{m} \pi^{1}\right)+\lambda\left(\pi^{1} \cdot \hat{\mathbf{k}}\right) \hat{\mathbf{x}}_{m}\right. \\
& +\frac{2 \underline{\mu} a_{1} a_{2} a_{3} h_{1} h_{2} h_{3}\left(\tau^{2}-1\right)}{\Lambda \Lambda^{\prime}}\left(\sum_{k=1}^{3} \frac{c_{1 k}^{1, k}}{h_{k} a_{k}^{2}}\right) \hat{\mathbf{x}}_{m} \\
& \left.+3 h_{1} h_{2} h_{3} \sum_{k=1}^{3} \frac{I_{1}^{k}}{h_{k}}\left(\underline{\mu}\left(c_{1 m}^{1, k} \hat{\mathbf{x}}_{k}-\delta_{k m} \mathbf{c}_{1}^{1, k}\right)+\lambda \tau^{2} c_{1 k}^{1, k} \hat{\mathbf{x}}_{m}\right)\right] \\
& +\frac{3\left(\tau^{2}+1\right)}{a_{m}}\left(a_{1} a_{2} a_{3} I_{1}^{m}-1\right) \mathbf{c}_{1}^{1, m}+\frac{3\left(\tau^{2}-1\right)}{a_{m}} \sum_{k=1}^{3} \frac{h_{m}}{h_{k}}\left(a_{1} a_{2} a_{3} I_{1}^{k}-\frac{a_{m}^{2}}{a_{k}^{2}}\right) c_{1 m}^{1, k} \hat{\mathbf{x}}_{k} \\
& +\frac{2\left(\tau^{2}-1\right)\left(\Lambda-a_{1}^{2}\right)\left(\Lambda-a_{2}^{2}\right)\left(\Lambda-a_{3}^{2}\right)}{\Lambda-\Lambda^{\prime}} \frac{h_{m} \hat{\mathbf{x}}_{m}}{a_{m}\left(\Lambda-a_{m}^{2}\right)}\left(a_{1} a_{2} a_{3} \Lambda I_{2}^{1}\right. \\
& \left.-\frac{a_{m}^{2}}{\Lambda}\right) \sum_{k=1}^{3} \frac{c_{1 k}^{1, k}}{h_{k}\left(\Lambda-a_{k}^{2}\right)} \\
& -\frac{2\left(\tau^{2}-1\right)\left(\Lambda^{\prime}-a_{1}^{2}\right)\left(\Lambda^{\prime}-a_{2}^{2}\right)\left(\Lambda^{\prime}-a_{3}^{2}\right)}{\Lambda-\Lambda^{\prime}} \frac{h_{m^{\prime}} \hat{\mathbf{x}}_{m}}{a_{m}\left(\Lambda^{\prime}-a_{m}^{2}\right)}\left(a_{1} a_{2} a_{3} \Lambda^{\prime} I_{2}^{2}\right. \\
& \left.\left.-\frac{a_{m}^{2}}{\Lambda^{\prime}}\right) \sum_{k=1}^{3} \frac{c_{1 k}^{1, k}}{h_{k}\left(\Lambda^{\prime}-a_{k}^{2}\right)}\right\} E_{1}^{m}(\mu) E_{1}^{m}(\nu)
\end{aligned}
$$




$$
\begin{aligned}
& \left.+\left(\frac{1}{a_{3}^{2}}-a_{1} a_{2} a_{3} I_{2}^{4}\right)\left(\frac{a_{1}^{2}}{h_{3}} c_{11}^{1,3}+\frac{a_{3}^{2}}{h_{1}} c_{13}^{1,1}\right) \hat{\mathbf{x}}_{3}\right) E_{1}^{1}(\mu) E_{1}^{1}(\nu) \\
& +\frac{3 \underline{\mu}\left(\tau^{2}-1\right)}{\sqrt{a_{1}^{2}-\mu^{2}} \sqrt{a_{1}^{2}-\nu^{2}}} \frac{h_{2}}{a_{2}}\left\{\left(\frac{1}{a_{1}^{2}}-a_{1} a_{2} a_{3} I_{2}^{3}\right)\left(\frac{a_{1}^{2}}{h_{2}} c_{11}^{1.2}+\frac{a_{2}^{2}}{h_{1}} c_{12}^{1.1}\right) \hat{\mathbf{x}}_{1}\right. \\
& \left.+\left(\frac{1}{a_{3}^{2}}-a_{1} a_{2} a_{3} I_{2}^{5}\right)\left(\frac{a_{2}^{2}}{h_{3}} c_{12}^{1,3}+\frac{a_{3}^{2}}{h_{2}} c_{13}^{1,2}\right) \hat{\mathbf{x}}_{3}\right) E_{1}^{2}(\mu) E_{1}^{2}(\nu) \\
& +\frac{3 \underline{\mu}\left(\tau^{2}-1\right)}{\sqrt{a_{1}^{2}-\mu^{2}} \sqrt{a_{1}^{2}-\nu^{2}}} \frac{h_{3}}{a_{3}}\left\{\left(\frac{1}{a_{1}^{2}}-a_{1} a_{2} a_{3} I_{2}^{4}\right)\left(\frac{a_{1}^{2}}{h_{3}} c_{11}^{1.3}+\frac{a_{3}^{2}}{h_{1}} c_{13}^{1,1}\right) \hat{\mathbf{x}}_{1}\right. \\
& \left.+\left(\frac{1}{a_{2}^{2}}-a_{1} a_{2} a_{3} I_{2}^{5}\right)\left(\frac{a_{2}^{2}}{h_{3}} c_{12}^{1,3}+\frac{a_{3}^{2}}{h_{2}} c_{13}^{1,2}\right) \hat{\mathbf{x}}_{2}\right\} E_{1}^{3}(\mu) E_{1}^{3}(\nu)=\mathbf{0} .
\end{aligned}
$$

In the form (92) we can use the orthogonality of the surface ellipsoidal harmonics $E_{0}^{1}(\mu) E_{0}^{1}(\nu)$ and $E_{1}^{k}(\mu) E_{1}^{k}(\nu), k=1,2,3$, immediately and obtain the coefficient

$$
\mathbf{c}_{0}^{1,1}=\mathbf{0}
$$

as well as a decoupled system of nine equations for the determination of the nine unknown coefficients $c_{1 k}^{1, n}, k, n=1,2,3$. In fact, by taking the inner product of the coefficient of $E_{1}^{m}(\mu) E_{1}^{m}(\nu)$ by $\hat{\mathbf{x}}_{m}, m=1,2,3$, we obtain a $3 \times 3$ system for the determination of $c_{1 m}^{1, m}, m=1,2,3$. The coefficients $c_{11}^{1,2}$ and $c_{12}^{1,1}$ are determined by the $2 \times 2$ system obtained from the $\hat{\mathbf{x}}_{2}$-component of the coefficient of $E_{1}^{1}(\mu) E_{1}^{1}(\nu)$ and the $\hat{\mathbf{x}}_{1}$-component of the coefficient of $E_{1}^{2}(\mu) E_{1}^{2}(\nu)$. Similarly, we obtain $c_{11}^{1.3}$ and $c_{13}^{1.1}$ by taking the inner product of $\hat{\mathbf{x}}_{3}$ with the coefficient of $E_{1}^{1}(\mu) E_{1}^{1}(\nu)$ and of $\hat{\mathbf{x}}_{1}$ with the coefficient of $E_{1}^{3}(\mu) E_{1}^{3}(\nu)$. Finally, the $2 \times 2$ system that determines $c_{12}^{1,3}$ and $c_{13}^{1,2}$ is obtained by taking the $\hat{\mathbf{x}}_{2}$-component of the coefficient of $E_{1}^{3}(\mu) E_{1}^{3}(\nu)$ and the $\hat{\mathbf{x}}_{3}$-component of the coefficient of $E_{1}^{2}(\mu) E_{1}^{2}(\nu)$. After we find and solve explicitly the above four systems we obtain the following expressions for the c's:

$$
c_{1 k}^{1, k}=\frac{1}{D h_{1} h_{2} h_{3}} \pi^{1} \otimes \hat{\mathbf{k}}: \tilde{J}_{k}, \quad k=1,2,3,
$$

and

$$
c_{1 k}^{1, n}=\frac{h_{m}}{3 h_{1} h_{2} h_{3}} \pi^{1} \otimes \hat{\mathbf{k}}: \tilde{W}_{k m}, \quad k \neq n,
$$

where

$$
\left.\begin{array}{l}
\tilde{J}_{k}(\rho)=\sum_{m=1}^{3}(-1)^{k+m+1} D_{m k}(\rho)\left(2 \hat{\mathbf{x}}_{m} \otimes \hat{\mathbf{x}}_{m}+\frac{\lambda}{\underline{\mu}} \tilde{I}\right), \\
\tilde{J}_{k}=\tilde{J}_{k}\left(a_{1}\right), \quad k=1,2,3
\end{array}\right\}
$$




$$
\begin{aligned}
& \left.\begin{array}{c}
D(\rho)=\operatorname{det}\left[\Theta_{n k}(\rho)\right] \\
D=D\left(a_{1}\right)
\end{array}\right\} \\
& \left.\begin{array}{c}
\Theta_{n k}(\rho)=\frac{3 \lambda I_{1}^{k}(\rho)-2(\lambda+\underline{\mu}) M_{n k}(\rho)}{h_{k}(\lambda+2 \underline{\mu})}+\frac{3 \delta_{n k}}{h_{k}}\left(\frac{2 \underline{\mu} I_{1}^{k}(\rho)}{\lambda+2 \underline{\mu}}-\frac{1}{a_{1} a_{2} a_{3}}\right), \\
\Theta_{n k}=\Theta_{n k}\left(a_{1}\right), \quad n, k=1,2,3,
\end{array}\right\} \\
& D_{m k}(\rho)=\text { the minor determinant of } D(\rho) \\
& \text { corresponding to the } m k \text {-entry, } \\
& D_{m k}=D_{m k}\left(a_{1}\right), \quad m, k=1,2,3 \text {, } \\
& M_{n k}(\rho)=\frac{1}{\Lambda-\Lambda^{\prime}}\left[\frac{\Lambda\left(\Lambda-a_{1}^{2}\right)\left(\Lambda-a_{2}^{2}\right)\left(\Lambda-a_{3}^{2}\right)}{\left(\Lambda-a_{n}^{2}\right)\left(\Lambda-a_{k}^{2}\right)} I_{2}^{1}(\rho)\right. \\
& \left.\left.-\frac{\Lambda^{\prime}\left(\Lambda^{\prime}-a_{1}^{2}\right)\left(\Lambda^{\prime}-a_{2}^{2}\right)\left(\Lambda^{\prime}-a_{3}^{2}\right)}{\left(\Lambda^{\prime}-a_{n}^{2}\right)\left(\Lambda^{\prime}-a_{k}^{2}\right)} I_{2}^{2}(\rho)\right],\right\} \\
& M_{n k}=M_{n k}\left(a_{1}\right), \quad n, k=1,2,3 \text {, } \\
& \tilde{W}_{k n}(\rho)=\frac{1}{\Omega_{k n}(\rho)}\left(\hat{\mathbf{x}}_{k} \otimes \hat{\mathbf{x}}_{n}+\hat{\mathbf{x}}_{n} \otimes \hat{\mathbf{x}}_{k}\right) \\
& \left.\tilde{W}_{k n}=\tilde{W}_{k n}\left(a_{1}\right), \quad k, n=1,2,3, k \neq n,\right\} \\
& \left.\begin{array}{l}
\Omega_{k n}(\rho)=\frac{1}{a_{1} a_{2} a_{3}}+\left(\tau^{2}-1\right)\left(a_{k}^{2}+a_{n}^{2}\right) I_{2}^{k+n}(\rho)-\tau^{2}\left(I_{1}^{k}(\rho)+I_{1}^{n}(\rho)\right), \\
\Omega_{k n}=\Omega_{k n}\left(a_{1}\right), \quad k, n=1,2,3, k \neq n .
\end{array}\right\}
\end{aligned}
$$

In terms of the given values of the c's, relations (81)-(87) for the $\beta$ 's yield

$$
\begin{aligned}
& \beta_{0}^{1,1}=-\frac{1}{D} \pi^{1} \otimes \hat{\mathbf{k}}: \sum_{k=1}^{3} \frac{1}{h_{k}} \tilde{J}_{k}, \\
& \beta_{1}^{1, k}=0, \quad k=1,2,3, \\
& \beta_{2}^{1,1}=\frac{3 \Lambda}{5 D h_{1}^{2} h_{2}^{2} h_{3}^{2}\left(\Lambda-\Lambda^{\prime}\right)} \pi^{1} \otimes \hat{\mathbf{k}}: \sum_{k=1}^{3}(-1)^{k+1} h_{k}\left(\Lambda^{\prime}-a_{k}^{2}\right) \tilde{J}_{k}, \\
& \beta_{2}^{1,2}=\frac{-3 \Lambda^{\prime}}{5 D h_{1}^{2} h_{2}^{2} h_{3}^{2}\left(\Lambda-\Lambda^{\prime}\right)} \pi^{1} \otimes \hat{\mathbf{k}}: \sum_{k=1}^{3}(-1)^{k+1} h_{k}\left(\Lambda-a_{k}^{2}\right) \tilde{J}_{k}, \\
& \beta_{2}^{1,3}=\frac{-1}{5 h_{1}^{2} h_{2}^{2} h_{3}^{2}} \pi^{1} \otimes \hat{\mathbf{k}}:\left(a_{1}^{2}+a_{2}^{2}\right) h_{1} h_{2} \tilde{W}_{12}^{\prime}, \\
& \beta_{2}^{1,4}=\frac{-1}{5 h_{1}^{2} h_{2}^{2} h_{3}^{2}} \pi^{1} \otimes \hat{\mathbf{k}}:\left(a_{1}^{2}+a_{3}^{2}\right) h_{1} h_{3} \tilde{W}_{13}, \\
& \beta_{2}^{1,5}=\frac{-1}{5 h_{1}^{2} h_{2}^{2} h_{3}^{2}} \pi^{1} \otimes \hat{\mathbf{k}}:\left(a_{2}^{2}+a_{3}^{2}\right) h_{2} h_{3} \tilde{W}_{23} .
\end{aligned}
$$


Finally, we substitute (93)-(95) and (103)-(109) into

$$
\Phi_{1}(\mathbf{r})=\pi^{1}(\hat{\mathbf{k}} \cdot \mathbf{r})+\mathbf{U}_{1}^{\text {cart }}(\mathbf{r})+\mathbf{R}^{e} U_{1}^{\text {ellip }}(\mathbf{r})
$$

and perform all the necessary calculations to conclude the first-order field

$$
\begin{aligned}
\Phi_{1}(\mathbf{r})= & \pi^{1} \otimes \hat{\mathbf{k}} \cdot \mathbf{r}+\pi^{1} \otimes \hat{\mathbf{k}}:{ }^{4} \tilde{Q}_{1}(\rho) \cdot \mathbf{r} \\
& +\pi^{1} \otimes \hat{\mathbf{k}}:\left[\tilde{P}(\rho)+{ }^{4} \tilde{Q}_{2}(\rho): \mathbf{r} \otimes \mathbf{r}\right] \otimes \mathbf{R}^{e},
\end{aligned}
$$

where

$$
\begin{aligned}
\mathbf{R}^{e}= & \left(\rho^{2}-\mu^{2}\right)^{-1 / 2}\left(\rho^{2}-\nu^{2}\right)^{-1 / 2} \frac{\rho}{h_{\rho}} \sum_{i=1}^{3} \frac{\hat{\mathbf{x}}_{i} \otimes \hat{\mathbf{x}}_{i}}{\rho^{2}-a_{1}^{2}+a_{i}^{2}} \cdot \mathbf{r}, \\
{ }^{4} \tilde{Q}_{1}(\rho)= & \sum_{n=1}^{3}\left[\sum_{\substack{k=1 \\
k \neq n}}^{3}\left(\frac{\left(\tau^{2}+1\right)}{2} I_{1}^{n}(\rho)+\frac{\left(\tau^{2}-1\right)}{2} I_{1}^{k}(\rho)\right) \tilde{W}_{k n} \otimes \hat{\mathbf{x}}_{k}\right. \\
& \left.+\frac{3 \tau^{2}}{D} \frac{I_{1}^{n}(\rho)}{h_{n}} \tilde{J}_{n} \otimes \hat{\mathbf{x}}_{n}+\frac{\tau^{2}-1}{D} \sum_{k=1}^{3} \frac{M_{n k}(\rho)}{h_{k}} \tilde{J}_{k} \otimes \hat{\mathbf{x}}_{n}\right] \otimes \hat{\mathbf{x}}_{n} \\
& -\frac{\tau^{2}-1}{2}\left[\left(a_{1}^{2}+a_{2}^{2}\right) I_{2}^{3}(\rho) \tilde{W}_{12} \otimes\left(\hat{\mathbf{x}}_{1} \otimes \hat{\mathbf{x}}_{2}+\hat{\mathbf{x}}_{2} \otimes \hat{\mathbf{x}}_{1}\right)\right. \\
& +\left(a_{1}^{2}+a_{3}^{2}\right) I_{2}^{4}(\rho) \tilde{W}_{13} \otimes\left(\hat{\mathbf{x}}_{1} \otimes \hat{\mathbf{x}}_{3}+\hat{\mathbf{x}}_{3} \otimes \hat{\mathbf{x}}_{1}\right) \\
& \left.+\left(a_{2}^{2}+a_{3}^{2}\right) I_{2}^{5}(\rho) \tilde{W}_{23} \otimes\left(\hat{\mathbf{x}}_{2} \otimes \hat{\mathbf{x}}_{3}+\hat{\mathbf{x}}_{3} \otimes \hat{\mathbf{x}}_{2}\right)\right], \\
\tilde{P}(\rho)= & \frac{\left(\tau^{2}-\right.}{2 D(\Lambda)\left(\rho^{2}-a_{1}^{2}\right)} \sum_{k=1}^{3} \frac{1}{h_{k}}\left[\frac{\left(\Lambda-a_{1}^{2}\right)\left(\Lambda-a_{2}^{2}\right)\left(\Lambda-a_{3}^{2}\right)}{\Lambda-a_{k}^{2}} \frac{1}{\left(E_{2}^{1}(\rho)\right)^{2}}\right. \\
& \left.-\frac{\left(\Lambda^{\prime}-a_{1}^{2}\right)\left(\Lambda^{\prime}-a_{2}^{2}\right)\left(\Lambda^{\prime}-a_{3}^{2}\right)}{\Lambda^{\prime}-a_{k}^{2}} \frac{1}{\left(E_{2}^{2}(\rho)\right)^{2}}\right] \tilde{J}_{k},
\end{aligned}
$$

and

$$
\begin{aligned}
{ }^{4} \tilde{Q}_{2}(\rho)= & \frac{\left(\tau^{2}-1\right)\left(\rho^{2}-a_{1}^{2}\right)}{2 D\left(\Lambda-\Lambda^{\prime}\right)} \sum_{n=1}^{3} \sum_{k=1}^{3} \frac{1}{h_{k}}\left[\frac{\left(\Lambda-a_{1}^{2}\right)\left(\Lambda-a_{2}^{2}\right)\left(\Lambda-a_{3}^{2}\right)}{\left(\Lambda-a_{k}^{2}\right)\left(\Lambda-a_{n}^{2}\right)} \frac{1}{\left(E_{2}^{1}(\rho)\right)^{2}}\right. \\
& \left.-\frac{\left(\Lambda^{\prime}-a_{1}^{2}\right)\left(\Lambda^{\prime}-a_{2}^{2}\right)\left(\Lambda^{\prime}-a_{3}^{2}\right)}{\left(\Lambda^{\prime}-a_{k}^{2}\right)\left(\Lambda^{\prime}-a_{n}^{2}\right)} \frac{1}{\left(E_{2}^{2}(\rho)\right)^{2}}\right] \tilde{J}_{k} \otimes \hat{\mathbf{x}}_{n} \otimes \hat{\mathbf{x}}_{n} \\
& -\left(\tau^{2}-1\right)\left(\rho^{2}-a_{1}^{2}\right)\left[\frac{1}{\left(E_{2}^{3}(\rho)\right)^{2}} \tilde{W}_{12} \otimes \hat{\mathbf{x}}_{1} \otimes \hat{\mathbf{x}}_{2}+\frac{1}{\left(E_{2}^{4}(\rho)\right)^{2}} \tilde{W}_{13} \otimes \hat{\mathbf{x}}_{1} \otimes \hat{\mathbf{x}}_{3}\right. \\
& \left.+\frac{1}{\left(E_{2}^{5}(\rho)\right)^{2}} \tilde{W}_{23} \otimes \hat{\mathbf{x}}_{2} \otimes \hat{\mathbf{x}}_{3}\right] .
\end{aligned}
$$


As indicated by the left superscript, ${ }^{4} \tilde{Q}_{n}(\rho), n=1,2$, is a tetradic.

Using the appropriate polarization, we obtain the exact form of the first-order lowfrequency field for an ellipsoidal cavity, which for $P$-incidence takes the form

$$
\begin{aligned}
\boldsymbol{\Phi}_{1}^{p}(\mathbf{r})= & \hat{\mathbf{k}} \otimes \hat{\mathbf{k}} \cdot \mathbf{r}+\hat{\mathbf{k}} \otimes \hat{\mathbf{k}}:{ }^{4} \tilde{Q}_{1}(\rho) \cdot \mathbf{r} \\
& +\hat{\mathbf{k}} \otimes \hat{\mathbf{k}}:\left[\tilde{P}(\rho)+{ }^{4} \tilde{Q}_{2}(\rho): \mathbf{r} \otimes \mathbf{r}\right] \otimes \mathbf{R}^{e},
\end{aligned}
$$

while for $S$ incidence it is

$$
\begin{aligned}
\Phi_{1}^{s}(\mathbf{r})= & \frac{1}{\tau} \hat{\mathbf{b}} \otimes \hat{\mathbf{k}} \cdot \mathbf{r}+\frac{1}{\tau} \hat{\mathbf{b}} \otimes \hat{\mathbf{k}}:{ }^{4} \tilde{Q}_{1}(\rho) \cdot \mathbf{r} \\
& +\frac{1}{\tau} \hat{\mathbf{b}} \otimes \hat{\mathbf{k}}:\left[\tilde{P}(\rho)+{ }^{4} \tilde{Q}_{2}(\rho): \mathbf{r} \otimes \mathbf{r}\right] \otimes \mathbf{R}^{e} .
\end{aligned}
$$

5. The scattering amplitudes and the scattering cross section. The leading low-frequency terms for the normalized spherical scattering amplitudes are obtained from (39)-(41) in the following form:

$$
\begin{aligned}
g_{r}(\hat{\mathbf{r}}, \hat{\mathbf{k}})=-\frac{i k^{3} \tau^{3}}{4 \pi}[(1- & \left.\left.2 \tau^{2}\right) \tilde{I}+2 \tau^{2} \hat{\mathbf{r}} \otimes \hat{\mathbf{r}}\right]:\left[V_{s} \pi^{0} \otimes \hat{\mathbf{r}}-\int_{S} \Phi_{1}\left(\mathbf{r}^{\prime}\right) \otimes \hat{\mathbf{n}}^{\prime} d s\left(\mathbf{r}^{\prime}\right)\right]+O\left(k^{4}\right) \\
g_{\theta}(\hat{\mathbf{r}}, \hat{\mathbf{k}})= & -\frac{i k^{3} \tau}{4 \pi}\left[\frac{V_{s}}{\tau} \pi^{0} \cdot \hat{\boldsymbol{\theta}}-2 \hat{\mathbf{r}} \otimes \hat{\theta}: \int_{S} \Phi_{1}\left(\mathbf{r}^{\prime}\right) \otimes \hat{\mathbf{n}}^{\prime} d s\left(\mathbf{r}^{\prime}\right)\right. \\
& \left.-\hat{\varphi} \cdot \int_{S} \boldsymbol{\Phi}_{1}\left(\mathbf{r}^{\prime}\right) \times \hat{\mathbf{n}}^{\prime} d s\left(\mathbf{r}^{\prime}\right)\right]+O\left(k^{4}\right) \\
g_{\varphi}(\hat{\mathbf{r}}, \hat{\mathbf{k}})= & -\frac{i k^{3} \tau}{4 \pi}\left[\frac{V_{s}}{\tau} \pi^{0} \cdot \hat{\varphi}-2 \hat{\mathbf{r}} \otimes \hat{\varphi}: \int_{S} \Phi_{1}\left(\mathbf{r}^{\prime}\right) \otimes \hat{\mathbf{n}}^{\prime} d s\left(\mathbf{r}^{\prime}\right)\right. \\
& \left.+\hat{\boldsymbol{\theta}} \cdot \int_{S} \Phi_{1}\left(\mathbf{r}^{\prime}\right) \times \hat{\mathbf{n}}^{\prime} d s\left(\mathbf{r}^{\prime}\right)\right]+O\left(k^{4}\right)
\end{aligned}
$$

where

$$
V_{s}=\frac{4 \pi}{3} a_{1} a_{2} a_{3}
$$

is the volume of the ellipsoidal cavity.

From (118)-(120) we observe that we only need to evaluate the dyadic integral

$$
\int_{S} \Phi_{1}\left(\mathbf{r}^{\prime}\right) \otimes \hat{\mathbf{n}}^{\prime} d s\left(\mathbf{r}^{\prime}\right)
$$

and its vector invariant.

Using the general form (111) for $\Phi_{1}$, where the $\mathbf{r}$ dependence is explicit, we conclude that

$$
\begin{aligned}
\int_{S} \boldsymbol{\Phi}_{1}\left(\mathbf{r}^{\prime}\right) \otimes \hat{\mathbf{n}}^{\prime} d s\left(\mathbf{r}^{\prime}\right)=\pi^{1} \otimes \hat{\mathbf{k}} \cdot \int_{S} \mathbf{r}^{\prime} \otimes \hat{\boldsymbol{\rho}}^{\prime} d s\left(\mathbf{r}^{\prime}\right) \\
+\pi^{1} \otimes \hat{\mathbf{k}}:\left[{ }^{4} \tilde{Q}_{1}\left(a_{1}\right) \cdot \int_{S} \mathbf{r}^{\prime} \otimes \hat{\boldsymbol{\rho}}^{\prime} d s\left(\mathbf{r}^{\prime}\right)+\tilde{P}\left(a_{1}\right) \otimes \int_{S} \frac{\hat{\boldsymbol{\rho}}^{\prime} \otimes \hat{\boldsymbol{\rho}}^{\prime}}{\sqrt{a_{1}^{2}-\mu^{\prime 2}} \sqrt{a_{1}^{2}-\nu^{\prime 2}}} d s\left(\mathbf{r}^{\prime}\right)\right. \\
\left.+{ }^{4} \tilde{Q}_{2}\left(a_{1}\right): \int_{S} \frac{\mathbf{r}^{\prime} \otimes \mathbf{r}^{\prime} \otimes \hat{\boldsymbol{\rho}}^{\prime} \otimes \hat{\boldsymbol{\rho}}^{\prime}}{\sqrt{a_{1}^{2}-\mu^{\prime 2}} \sqrt{a_{1}^{2}-\nu^{\prime 2}}} d s\left(\mathbf{r}^{\prime}\right)\right]
\end{aligned}
$$


Introducing a combination of ellipsoidal with spherical coordinates, we were able to evaluate, in [8], the following integrals:

$$
\begin{gathered}
\int_{S} \mathbf{r}^{\prime} \otimes \hat{\boldsymbol{\rho}}^{\prime} d s\left(\mathbf{r}^{\prime}\right)=V_{s} \tilde{I}, \\
\int_{S} \frac{\hat{\boldsymbol{\rho}}^{\prime} \otimes \hat{\boldsymbol{\rho}}^{\prime}}{\sqrt{a_{1}^{2}-\mu^{\prime 2}} \sqrt{a_{1}^{2}-\nu^{\prime 2}}} d s\left(\mathbf{r}^{\prime}\right)=3 V_{s} \sum_{k=1}^{3} I_{1}^{k} \hat{\mathbf{x}}_{k} \otimes \hat{\mathbf{x}}_{k} .
\end{gathered}
$$

From expressions (114) and (115) we see immediately that the tensors $\tilde{P}$ and ${ }^{4} \tilde{Q}_{2}$ vanish at $\rho=a_{1}$ and therefore there is no need to evaluate the last integral in (122).

By virtue of (123) and (124) and the above observation, Equation (122) implies

$$
\int_{S} \Phi_{1}\left(\mathbf{r}^{\prime}\right) \otimes \hat{\mathbf{n}}^{\prime} d s\left(\mathbf{r}^{\prime}\right)=\frac{4 \pi}{3} a_{1} a_{2} a_{3}\left[\pi^{1} \otimes \hat{\mathbf{k}}+\pi^{1} \otimes \hat{\mathbf{k}}:{ }^{4} \tilde{Q}_{1}\left(a_{1}\right)\right] .
$$

The vector invariant of (125) gives

$$
\int_{S} \boldsymbol{\Phi}_{1}\left(\mathbf{r}^{\prime}\right) \times \hat{\mathbf{n}}^{\prime} d s\left(\mathbf{r}^{\prime}\right)=\frac{4 \pi}{3} a_{1} a_{2} a_{3}\left[\pi^{1} \times \hat{\mathbf{k}}+\mathbf{g}_{1}\left(a_{1}\right)\right],
$$

where

$\mathbf{g}_{1}\left(a_{1}\right)=\pi^{1} \otimes \hat{\mathbf{k}}:\left[h_{3}^{2} I_{2}^{3} \tilde{W}_{12} \otimes \hat{\mathbf{x}}_{1} \times \hat{\mathbf{x}}_{2}+h_{2}^{2} I_{2}^{4} \tilde{W}_{13} \otimes \hat{\mathbf{x}}_{1} \times \hat{\mathbf{x}}_{3}+h_{1}^{2} I_{2}^{5} \tilde{W}_{23} \otimes \hat{\mathbf{x}}_{2} \times \hat{\mathbf{x}}_{3}\right]$.

Finally, we insert (125) and (126) into (118)-(120) and derive the expressions

$$
\begin{aligned}
g_{r}(\hat{\mathbf{r}}, \hat{\mathbf{k}})= & \frac{i k^{3} \tau^{3}}{3} a_{1} a_{2} a_{3}\left\{\left(1-2 \tau^{2}\right)\left[\pi^{1} \cdot \hat{\mathbf{k}}+\pi^{1} \otimes \hat{\mathbf{k}}:{ }^{4} \tilde{Q}_{1}\left(a_{1}\right): \tilde{I}\right]\right. \\
& \left.-\pi^{0} \cdot \hat{\mathbf{r}}+2 \tau^{2}\left[\pi^{1} \otimes \hat{\mathbf{k}}+\pi^{1} \otimes \hat{\mathbf{k}}:{ }^{4} \tilde{Q}_{1}\left(a_{1}\right)\right]: \hat{\mathbf{r}} \otimes \hat{\mathbf{r}}\right\}+O\left(k^{4}\right), \\
g_{\theta}(\hat{\mathbf{r}}, \hat{\mathbf{k}})= & \frac{i k^{3}}{3} a_{1} a_{2} a_{3}\left\{\tau \hat{\varphi} \cdot\left(\pi^{1} \times \hat{\mathbf{k}}+\mathbf{g}_{1}\left(a_{1}\right)\right)\right. \\
& \left.-\pi^{0} \cdot \hat{\boldsymbol{\theta}}+2 \tau\left[\pi^{1} \otimes \hat{\mathbf{k}}+\pi^{1} \otimes \hat{\mathbf{k}}:{ }^{4} \tilde{Q}_{1}\left(a_{1}\right)\right]: \hat{\boldsymbol{\theta}} \otimes \hat{\mathbf{r}}\right\}+O\left(k^{4}\right), \\
g_{\varphi}(\hat{\mathbf{r}}, \hat{\mathbf{k}})= & \frac{i k^{3}}{3} a_{1} a_{2} a_{3}\left\{-\tau \hat{\boldsymbol{\theta}} \cdot\left(\pi^{1} \times \hat{\mathbf{k}}+\mathbf{g}_{1}\left(a_{1}\right)\right)\right. \\
& \left.-\pi^{0} \cdot \hat{\varphi}+2 \tau\left[\pi^{1} \otimes \hat{\mathbf{k}}+\pi^{1} \otimes \hat{\mathbf{k}}:{ }^{4} \tilde{Q}_{1}\left(a_{1}\right)\right]: \hat{\varphi} \otimes \hat{\mathbf{r}}\right\}+O\left(k^{4}\right) .
\end{aligned}
$$

When the incident wave is a longitudinal wave we substitute $\pi^{0}=\pi^{1}=\hat{\mathbf{k}}$ into (128)-(130), while for an incident transverse wave we substitute $\pi^{0}=\tau \pi^{1}=\hat{\mathbf{b}}$.

The scattering cross section for $P$-incidence is given by (26)

$$
\begin{aligned}
\boldsymbol{\sigma}^{p}= & \frac{\tau^{3} k^{4}}{60 \pi}\left\{\frac{5\left(\tau^{3}+2\right)}{\tau^{2}} V_{s}^{2}+\left(28 \tau^{5}-40 \tau^{3}+15 \tau-8\right)\left|\int_{S} \boldsymbol{\Phi}_{1}\left(\mathbf{r}^{\prime}\right) \cdot \hat{\mathbf{n}}^{\prime} d s\left(\mathbf{r}^{\prime}\right)\right|^{2}\right. \\
& \left.-10\left|\int_{S} \boldsymbol{\Phi}_{1}\left(\mathbf{r}^{\prime}\right) \times \hat{\mathbf{n}}^{\prime} d s\left(\mathbf{r}^{\prime}\right)\right|^{2}+4\left(\tau^{5}+4\right)\left\|\int_{S} \boldsymbol{\Phi}_{1}\left(\mathbf{r}^{\prime}\right) \otimes \hat{\mathbf{n}}^{\prime} d s\left(\mathbf{r}^{\prime}\right)\right\|^{2}\right\}+O\left(k^{6}\right) .
\end{aligned}
$$


The integrals that appear in (131) assume the values

$$
\left|\int_{S} \Phi_{1}\left(\mathbf{r}^{\prime}\right) \cdot \hat{\mathbf{n}}^{\prime} d s\left(\mathbf{r}^{\prime}\right)\right|^{2}=V_{s}^{2}\left|\pi^{1} \cdot \hat{\mathbf{k}}+\pi^{1} \otimes \hat{\mathbf{k}}: \tilde{J}\right|^{2},
$$

where

$$
\begin{gathered}
\tilde{J}=\frac{\tau^{2}-1}{D} \sum_{n=1}^{3} \sum_{k=1}^{3} \frac{M_{n k}}{h_{k}} \tilde{J}_{k}+\frac{3 \tau^{2}}{D} \sum_{n=1}^{3} \frac{I_{1}^{n}}{h_{n}} \tilde{J}_{n}, \\
\left|\int_{S} \Phi_{1}\left(\mathbf{r}^{\prime}\right) \times \hat{\mathbf{n}}^{\prime} d s\left(\mathbf{r}^{\prime}\right)\right|^{2}=V_{s}^{2}\left|\pi^{1} \times \hat{\mathbf{k}}+\mathbf{g}_{1}\left(a_{1}\right)\right|^{2}, \\
\left\|\int_{S} \Phi_{1}\left(\mathbf{r}^{\prime}\right) \otimes \hat{\mathbf{n}}^{\prime} d s\left(\mathbf{r}^{\prime}\right)\right\|^{2}=V_{s}^{2}\left\|\pi^{1} \otimes \hat{\mathbf{k}}+\pi^{1} \otimes \hat{\mathbf{k}}:{ }^{4} \tilde{Q}_{1}\left(a_{1}\right)\right\|^{2} .
\end{gathered}
$$

Consequently, the scattering cross section for $P$-incidence is given by

$$
\begin{aligned}
\boldsymbol{\sigma}^{p}= & \frac{V_{s}^{2} k^{4}}{60 \pi}\left[5 \tau\left(\tau^{3}+2\right)-10 \tau^{3}\left|\mathbf{g}_{1}\left(a_{1}\right)\right|^{2}+\tau^{3}\left(28 \tau^{5}-40 \tau^{3}+15 \tau-8\right)|1+\hat{\mathbf{k}} \otimes \hat{\mathbf{k}}: \tilde{J}|^{2}\right. \\
& \left.+4 \tau^{3}\left(\tau^{5}+4\right)\left\|\hat{\mathbf{k}} \otimes \hat{\mathbf{k}}+\hat{\mathbf{k}} \otimes \hat{\mathbf{k}}:{ }^{4} \tilde{Q}_{1}\left(a_{1}\right)\right\|^{2}\right]+O\left(k^{6}\right)
\end{aligned}
$$

while the scattering cross section for $S$-incidence is given by

$$
\begin{aligned}
& \boldsymbol{\sigma}^{s}=\frac{V_{s}^{2} k^{4}}{60 \pi}\left[5 \tau\left(\tau^{3}+2\right)+\left(28 \tau^{5}-40 \tau^{3}+15 \tau-8\right)|\hat{\mathbf{b}} \otimes \hat{\mathbf{k}}: \tilde{J}|^{2}-10 \tau\left|\hat{\mathbf{b}} \times \hat{\mathbf{k}}+\tau \mathbf{g}_{1}\left(a_{1}\right)\right|^{2}\right. \\
& \left.\quad+4 \tau\left(\tau^{5}+4\right)\left\|\hat{\mathbf{b}} \otimes \hat{\mathbf{k}}+\hat{\mathbf{b}} \otimes \hat{\mathbf{k}}:{ }^{4} \tilde{Q}_{1}\left(a_{1}\right)\right\|^{2}\right]+O\left(k^{6}\right)
\end{aligned}
$$

\section{Special shapes}

A. Spheroids. A prolate spheroid is obtained whenever $a_{1}>a_{2}=a_{3}$, while the case of an oblate spheroid corresponds to $a_{1}<a_{2}=a_{3}$. The elliptic integrals can be evaluated in closed form for spheroids:

$$
\begin{aligned}
& I_{0}^{1}(\rho)=\frac{1}{h_{3}} \begin{cases}\frac{1}{2} \ln \left(\frac{\rho+h_{3}}{\rho-h_{3}}\right), & a_{1}>a_{2}, \\
\frac{1}{i} \tan ^{-1}\left(\frac{i h_{3}}{\rho}\right), & a_{1}<a_{2},\end{cases} \\
& I_{1}^{1}(\rho)=\frac{1}{h_{3}^{2}}\left(I_{0}^{1}(\rho)-\frac{1}{\rho}\right), \\
& I_{1}^{2}(\rho)=I_{1}^{3}(\rho)=-\frac{1}{2 h_{3}^{2}}\left(I_{0}^{1}(\rho)-\frac{\rho}{\rho^{2}-h_{3}^{2}}\right),
\end{aligned}
$$




$$
\begin{aligned}
& I_{2}^{1}(\rho)=\frac{9}{4 h_{3}^{4}}\left(I_{0}^{1}(\rho)-\frac{3 \rho}{3 \rho^{2}-h_{3}^{2}}\right), \\
& I_{2}^{2}(\rho)=I_{2}^{5}(\rho)=\frac{3}{8 h_{3}^{4}}\left(I_{0}^{1}(\rho)-\frac{\rho\left(3 \rho^{2}-5 h_{3}^{2}\right)}{3\left(\rho^{2}-h_{3}^{2}\right)}\right), \\
& I_{2}^{3}(\rho)=I_{2}^{4}(\rho)=-\frac{3}{2 h_{3}^{4}}\left(I_{0}^{1}(\rho)-\frac{3 \rho^{2}-2 h_{3}^{2}}{3 \rho\left(\rho^{2}-h_{3}^{2}\right)}\right),
\end{aligned}
$$

where

$$
\rho=\left\{\begin{array}{l}
h_{3} \cosh \omega \\
h_{3} i \sinh \omega
\end{array}= \begin{cases}\sqrt{a_{1}^{2}-a_{2}^{2}} \cosh \omega, & a_{1}>a_{2}, \\
\sqrt{a_{2}^{2}-a_{1}^{2}} \sinh \omega, & a_{1}<a_{2},\end{cases}\right.
$$

and $(\omega, \theta, \varphi)$ are the spheroidal coordinates, which are related to the Cartesian coordinates $\left(x_{1}, x_{2}, x_{3}\right)$ by

$$
\begin{aligned}
& x_{1}=\rho \cos \theta, \quad \omega \in[0,+\infty), \\
& x_{2}=\sqrt{\rho^{2}-h_{3}^{2}} \sin \theta \cos \varphi, \quad \theta \in[0, \pi], \\
& x_{3}=\sqrt{\rho^{2}-h_{3}^{2}} \sin \theta \sin \varphi, \quad \varphi \in[0,2 \pi) .
\end{aligned}
$$

For $\rho=a_{1}$ we obtain

$$
I_{0}^{1}=\frac{1}{a_{2}} \begin{cases}{\left[\left(\frac{a_{1}}{a_{2}}\right)^{2}-1\right]^{-1 / 2} \cosh ^{-1}\left(\frac{a_{1}}{a_{2}}\right),} & a_{1}>a_{2}, \\ {\left[1-\left(\frac{a_{1}}{a_{2}}\right)^{2}\right]^{-1 / 2} \cos ^{-1}\left(\frac{a_{1}}{a_{2}}\right),} & a_{1}>a_{2},\end{cases}
$$

and through (139)-(143) all the other elliptic integrals can be expressed as functions of the ratio $a_{1} / a_{2}$, whenever $\rho=a_{1}$.

Having the values of the elliptic integrals, we can substitute them in the corresponding expressions and obtain the results for an oblate or a prolate spheroid, as the case may be.

B. Needle and disc. The needle-shaped scatterer can be approximated by a prolate spheroid where $a_{1} \gg a_{2}=a_{3}$. In this case

$$
I_{0}^{1} \sim \frac{1}{a_{2}} \frac{\ln 2\left(a_{1} / a_{2}\right)}{\left(a_{1} / a_{2}\right)}, \quad \frac{a_{1}}{a_{2}} \rightarrow+\infty .
$$

In the case where $a_{1} \ll a_{2}=a_{3}$, the oblate spheroid takes the shape of a circular disc and

$$
I_{0}^{1} \sim \frac{\pi}{2 a_{2}}, \quad \frac{a_{1}}{a_{2}} \rightarrow 0+.
$$


C. Sphere. The sphere is the shape that corresponds to radial symmetry and comes out of the case where $a_{1}=a_{2}=a_{3}=a$. The elliptic integrals assume the following values:

$$
\begin{aligned}
& I_{0}^{1}(\rho)=\frac{1}{\rho}, \\
& I_{1}^{n}(\rho)=\frac{1}{3 \rho^{3}}, \quad n=1,2,3, \\
& I_{2}^{n}(\rho)=\frac{1}{5 \rho^{5}}, \quad n=1,2,3,4,5 .
\end{aligned}
$$

We also obtain $\rho=r, \mu=\nu=0$, and $\Lambda=\Lambda^{\prime}=a^{2}$. In order to evaluate the undetermined forms in the various expressions it is enough to approximate the sphere, say by a prolate spheroid, setting $a_{1}=a(1+\varepsilon), \varepsilon>0, a_{2}=a_{3}=a$, and obtain the case of a sphere in the limit as $\varepsilon \rightarrow 0+$.

7. Physical implications. The physical interpretation of the mathematical problem analyzed in this work involves a plane harmonic elastic wave, which could be either a longitudinal or a transverse wave, that propagates in the three-dimensional Euclidean space where there exists a cavity with the shape of a general triaxial ellipsoid. The existence of the ellipsoidal cavity, which is arbitrarily oriented with respect to the propagation vector of the incident plane wave, disturbs the incident wave, and as a result a much more complicated wave field is established. In fact, due to the linearity of the problem, a secondary "scattered" wave is radiated outward from the cavity which is superposed on the existing incident wave. On the surface of the ellipsoidal cavity the total (incident plus scattered) field should have zero surface traction, while at large distance from the scattering region the incident field should be prominent and the scattered field should die out at a given rate prescribed by the radiation conditions.

The case where the scattering region is a rigid ellipsoid, a physical case which is characterized by the vanishing of the displacement field on the surface of the ellipsoid, was studied in [8]. It turns out that, from the analytical point of view, the cavity problem is much harder than the rigid problem; this is due to the complexity of the boundary condition that describes the stress on the surface of the ellipsoid.

Problems of longitudinal as well as transverse incident waves are analyzed simultaneously by introducing a generalized polarization vector given by Equation (32).

Since the longitudinal wave is faster than the transverse wave, two wave numbers are involved in the problem. In our low-frequency analysis we use as parameters the wave number $k$ of the slower transverse wave and the ratio $\tau$ of the wave number of the longitudinal to the wave number of the transverse wave. With this choice of parameters $k$ should be much less than the maximum semiaxis $a_{1}$ of the ellipsoid, and of course $\tau \in(0,1)$.

The zeroth-order low-frequency approximation is a constant vector which coincides with the unit propagation vector in the case of longitudinal incidence and with the unit polarization vector in the case of transverse incidence. This behaviour is not shared with the rigid scatterer, where the zeroth-order approximation was dependent on the observation point [8]. 
The real difficulty of the problem is focused on the evaluation of the first-order low-frequency approximation, which demands many mathematical techniques, and it is given by (116) for longitudinal incidence and by (117) for transverse incidence. The first-order approximation is expressed via the second-rank tensor $\tilde{P}$ and the fourth-rank tensors ${ }^{4} \tilde{Q}_{1},{ }^{4} Q_{2}$ given by Formulae (113)-(115). These tensors involve only the semiaxes $a_{1}, a_{2}, a_{3}$ (geometric characteristics), the Lamé constants $\lambda, \underline{\mu}$ (physical characteristics), and the ellipsoidal coordinate $\rho$, which determines the ellipsoidal coordinate surface that passes through the observation point $\mathbf{r}$. The dependence of the first-order approximation on the propagation vector, the polarization vector, and the observation point is explicitly given in (116) and (117). There are terms independent of $\mathbf{r}$, terms dependent on $\mathbf{r}$, and terms dependent on $\mathbf{r} \otimes \mathbf{r}$. As in the case of the rigid ellipsoid, there are a Cartesian part and an ellipsoidal part, which is directed along the outward normal direction $\mathbf{R}^{e}$ and vanishes on the surface of the ellipsoid since the tensors $\tilde{P}$ and ${ }^{4} \tilde{Q}_{2}$ vanish for $\rho=a_{1}$. Since the tensors $\tilde{P},{ }^{4} \tilde{Q}_{1}$, and ${ }^{4} \tilde{Q}_{2}$ are proportional to $\tau^{2}-1$, it follows that the farther apart the phase velocities of the longitudinal and transverse waves, the more prominent the first-order approximation. On the other hand, in the limiting case, where $\tau^{2} \rightarrow 1-$, we obtain the weakest form of the first-order approximation, which corresponds to the case of an acoustic rigid scatterer.

The normalized spherical scattering amplitudes $g_{r}, g_{\theta}, g_{\varphi}$ are the dimensionless quantities that determine the strength of the scattered field, far away from the scattering region, along the radial direction of $r$ and the tangential (to a sphere) directions of $\theta$ and $\varphi$, respectively. Their physical significance is due to the fact that the square of their magnitude is proportional to the energy density function; i.e., $\left|g_{r}(\hat{\mathbf{r}}, \hat{\mathbf{k}})\right|^{2}$ specifies the energy density that is scattered in the direction $\hat{\mathbf{r}}$ for incidence in the direction $\hat{\mathbf{k}}$. The values of $g_{r}, g_{\theta}, g_{\varphi}$ are given by (128)-(130). It is observed that they are all proportional to the volume of the ellipsoid; their leading low-frequency term is purely imaginary and of order $k^{3}$. The corresponding scattering amplitudes for the rigid ellipsoid have leading low-frequency terms of order $k$ [8].

The scattering cross section, which determines the total energy that the ellipsoidal cavity diverts from the incident wave and radiates in all directions, is given by Eq. (136) for longitudinal incidence and by Eq. (137) for transverse incidence. The scattering cross section is proportional to the square of the volume of the scattering region, and its leading low-frequency term is of order $k^{4}$. Comparing this result with the corresponding result for the rigid ellipsoid [8], we observe that the leading low-frequency term of the scattering cross section for the rigid case is independent of $k$. Consequently, in the low-frequency approximation a rigid ellipsoid scatters much more energy than an ellipsoidal cavity.

All the analytical results given in the present work are expressed in a form suitable for numerical calculations. In fact, they are all reduced to numerical values of given functions. It is of interest to investigate the special cases of geometric and physical degeneracy obtained in Sec. 6.

8. Appendix: Ellipsoidal harmonics. The interior ellipsoidal harmonics of degree $n$ are given by the Lamé products

$$
\mathbb{E}_{n}^{m}(\rho, \mu, \nu)=E_{n}^{m}(\rho) E_{n}^{m}(\mu) E_{n}^{m}(\nu)
$$

for $m=1,2, \ldots, 2 n+1$, where $E_{n}^{m}$ is the Lamé function of the first kind. 
Similarly, the exterior ellipsoidal harmonics of degree $n$ are given by

$$
\mathbb{F}_{n}^{m}(\rho, \mu, \nu)=F_{n}^{m}(\rho) E_{n}^{m}(\mu) E_{n}^{m}(\nu)
$$

for $m=1,2, \ldots, 2 n+1$, where $F_{n}^{m}$ are the Lamé functions of the second kind. These are related to $E_{n}^{m}(\rho)$ by the formula

$$
F_{n}^{m}(\rho)=(2 n+1) E_{n}^{m}(\rho) I_{n}^{m}(\rho),
$$

where the functions

$$
I_{n}^{m}(\rho)=\int_{\rho}^{+\infty} \frac{d u}{\left[E_{n}^{m}(u)\right]^{2} \sqrt{u^{2}-h_{2}^{2}} \sqrt{u^{2}-h_{3}^{2}}}
$$

express elliptic integrals.

There are $2 n+1$ linearly independent ellipsoidal harmonic functions of degree $n$ corresponding to the $2 n+1$ spherical harmonics of degree $n$. The Lamé functions $E_{n}^{m}(\rho)$ correspond to the radial factor $r^{n}$ of the interior spherical harmonics of degree $n$, while the Lamé functions $F_{n}^{m}(\rho)$ correspond to the radial factor $r^{-n-1}$ of the exterior spherical harmonics of degree $n$. In this work we only use the ellipsoidal harmonics of degree 0,1 , and 2, which are given in the sequel in their ellipsoidal as well as their Cartesian form.

$$
\begin{aligned}
& \mathbb{E}_{0}^{1}(\rho, \mu, \nu)=1 \\
& \mathbb{E}_{1}^{1}(\rho, \mu, \nu)=\rho \mu \nu \\
& =x_{1} h_{2} h_{3} \\
& \mathbb{E}_{1}^{2}(\rho, \mu, \nu)=\sqrt{\rho^{2}-h_{3}^{2}} \sqrt{\mu^{2}-h_{3}^{2}} \sqrt{h_{3}^{2}-\nu^{2}} \\
& =x_{2} h_{1} h_{3} \\
& \mathbb{E}_{1}^{3}(\rho, \mu, \nu)=\sqrt{\rho^{2}-h_{2}^{2}} \sqrt{h_{2}^{2}-\mu^{2}} \sqrt{h_{2}^{2}-\nu^{2}} \\
& =x_{3} h_{1} h_{2} \\
& \mathbb{E}_{2}^{1}(\rho, \mu, \nu)=\left(\rho^{2}-a_{1}^{2}+\Lambda\right)\left(\mu^{2}-a_{1}^{2}+\Lambda\right)\left(\nu^{2}-a_{1}^{2}+\Lambda\right) \\
& =\left(\Lambda-a_{1}^{2}\right)\left(\Lambda-a_{2}^{2}\right)\left(\Lambda-a_{3}^{2}\right)\left(\sum_{k=1}^{3} \frac{x_{k}^{2}}{\Lambda-a_{k}^{2}}+1\right) \\
& \mathbb{E}_{2}^{2}(\rho, \mu, \nu)=\left(\rho^{2}-a_{1}^{2}+\Lambda^{\prime}\right)\left(\mu^{2}-a_{1}^{2}+\Lambda^{\prime}\right)\left(\nu^{2}-a_{1}^{2}+\Lambda^{\prime}\right) \\
& =\left(\Lambda^{\prime}-a_{1}^{2}\right)\left(\Lambda^{\prime}-a_{2}^{2}\right)\left(\Lambda^{\prime}-a_{3}^{2}\right)\left(\sum_{k=1}^{3} \frac{x_{k}^{2}}{\Lambda^{\prime}-a_{k}^{2}}+1\right) \\
& \mathbb{E}_{2}^{3}(\rho, \mu, \nu)=\mathbb{E}_{1}^{1}(\rho, \mu, \nu) \mathbb{E}_{1}^{2}(\rho, \mu, \nu) \\
& =x_{1} x_{2} h_{1} h_{2} h_{3}^{2} \\
& \mathbb{E}_{2}^{4}(\rho, \mu, \nu)=\mathbb{E}_{1}^{1}(\rho, \mu, \nu) \mathbb{E}_{1}^{3}(\rho, \mu, \nu) \\
& =x_{1} x_{3} h_{1} h_{2}^{2} h_{3} \\
& \mathbb{E}_{2}^{5}(\rho, \mu, \nu)=\mathbb{E}_{1}^{2}(\rho, \mu, \nu) \mathbb{E}_{1}^{3}(\rho, \mu, \nu) \\
& =x_{2} x_{3} h_{1}^{2} h_{2} h_{3}
\end{aligned}
$$


where $\Lambda, \Lambda^{\prime}$ are the two roots of the equation

$$
\sum_{i=1}^{3} \frac{1}{\Lambda-a_{i}^{2}}=0
$$

The exterior ellipsoidal harmonics of degree 0,1 , and 2 are given by (A.2) when (A.3)-(A.13) are used. The Lamé functions of degree 0,1 , and 2 that appear in expression (A.4) for the elliptic integrals $I_{n}^{m}(\rho)$ are

$$
\begin{aligned}
E_{0}^{1}(\rho) & =1, \\
E_{1}^{m}(\rho) & =\sqrt{\rho^{2}-a_{1}^{2}+a_{m}^{2}}, \quad m=1,2,3, \\
E_{2}^{1}(\rho) & =\rho^{2}-a_{1}^{2}+\Lambda, \\
E_{2}^{2}(\rho) & =\rho^{2}-a_{1}^{2}+\Lambda^{\prime}, \\
E_{2}^{3}(\rho) & =\rho \sqrt{\rho^{2}-h_{3}^{2}}, \\
E_{2}^{4}(\rho) & =\rho \sqrt{\rho^{2}-h_{2}^{2}}, \\
E_{2}^{5}(\rho) & =\sqrt{\rho^{2}-h_{2}^{2}} \sqrt{\rho^{2}-h_{3}^{2}} .
\end{aligned}
$$

For a detailed analysis of the ellipsoidal harmonic functions and the relative useful properties see $[2,10]$.

The set of functions

$$
\left\{E_{n}^{m}(\mu) E_{n}^{m}(\nu): n=0,1,2, \ldots ; m=1,2,3, \ldots, 2 n+1\right\}
$$

forms a complete orthogonal set of surface harmonics on the surface of the ellipsoid

$$
\frac{x_{1}^{2}}{\rho^{2}}+\frac{x_{2}^{2}}{\rho^{2}-h_{3}^{2}}+\frac{x_{3}^{2}}{\rho^{2}-h_{2}^{2}}=1,
$$

which coincides with the surface of the scatterer whenever $\rho=a_{1}$.

Acknowledgment. The authors want to thank the Greek Ministry of Research and Technology for partially supporting the present work.

\section{REFERENCES}

[1] P. J. Barratt and W. D. Collins, The scattering cross section of an obstacle in an elastic solid for plane harmonic waves, Proc. Cambridge Philos. Soc. 61, 969 (1965)

[2] G. Dassios, Convergent low-frequency expansions for penetrable scatterers, Doctoral Dissertation, University of Illinois, Chicago (1975)

[3] G. Dassios, Convergent low-frequency expansions for penetrable scatterers, J. Math. Phys. 18, 126 (1977)

[4] G. Dassios, Second-order low-frequency scattering by the soft ellipsoid, SIAM J. Appl. Math. 38, 373 (1980)

[5] G. Dassios, Low-frequency scattering theory for a penetrable body with an impenetrable core, SIAM J. Appl. Math. 42, 272 (1982)

[6] G. Dassios, Scattering of acoustic waves by a coated pressure release ellipsoid, J. Acoust. Soc. Amer. 70, 176 (1981)

[7] G. Dassios and K. Kiriaki, The low-frequency theory of elastic wave scattering, Quart. Appl. Math. 42 , 225-248 (1984) 
[8] G. Dassios and K. Kiriaki, The rigid ellipsoid in the presence of a low frequency elastic wave, Quart Appl. Math. 43, 435-456 (1986)

[9] N. G. Einspruch, E. J. Witterholt, and R. Truell, Scattering of a plane transverse wave by a spherical obstacle in an elastic medium, J. Appl. Phys. 31, 806-818 (1960)

[10] E. W. Hobson, The theory of spherical and ellipsoidal harmonics, Chelsea (1955)

[11] V. D. Kupradze, Dynamical problems in elasticity, in Progress in solid mechanics III. North-Holland, Amsterdam (1963)

[12] E. G. Lawrence, Diffraction of elastic waves by a rigid ellipsoid, Quart. J. Mech. Appl. Math. XXV, 161 (1972)

[13] L. Solomon, Elasticité Linéaire, Masson (1968)

[14] V. Twersky, Certain transmission and reflection theorems, J. Appl. Phys. 25, 859 (1954)

[15] C. Truesdell, Mechanics of Solids II, in Encyclopedia of Physics, Vol. VI a/2, Springer-Verlag (1972)

[16] C. F. Ying and R. Truell, Scattering of a plane longitudinal wave by a spherical obstacle in an isotropically elastic solid, J. Appl. Phys. 27, 1086 (1956) 